CWP-144

DOE/ER/14079-31

March 1994

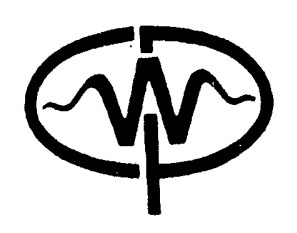

\title{
Body-Wave Radiation Patterns and AVO in Transversely Isotropic Media
}

\author{
Ilya Tsvankin
}

\begin{abstract}
DISCLAIMER
This report was prepared as an account of work sponsored by an agency of the United States This report was prepared as an States Government nor any agency thereof, nor any of their Government. Neither the United States Government nor any agency thereor, nor any of their employees, makes any warranty, express or implied, or assumes any legal liability or responsi-
bility for the accuracy, completeness, or usefulness of any information, apparatus, product, or process disclosed, or represents that its use would not infringe privately owned rights. Reference herein to any specific commercial product, process, or service by trade name, trademark, manufacturer, or otherwise dhe United States Government or any agency thereof. The views and opinions of authors expressed herein do not necessarily state or reflect those of the United States Government or any agency thereof.
\end{abstract}

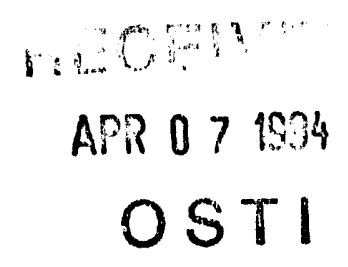

Center for Wave Phenomena

Colorado School of Mines

Golden, Colorado 80401

$303 / 273-3557$ 


\title{
Body-wave radiation patterns and AVO in transversely isotropic media
}

\author{
I. Tsvankin
}

\begin{abstract}
It is well known that the angular dependence of reflection coefficients may be significantly distorted in the presence of elastic anisotropy. However, the influence of anisotropy on amplitude-versus-offset analysis (AVO) is not limited to reflection coefficients. AVO signatures (e.g., AVO gradient) in anisotropic media are also distorted by the redistribution of energy along the wavefront of the wave travelling down to the reflector and back up to the surface. Significant anisotropy above the target horizon may be rather typical of sand-shale sequences commonly encountered in AVO analysis.

Here, I examine the influence of $\mathrm{P}$ - and S-wave radiation patterns on AVO in the most common anisotropic model - transversely isotropic media. A concise analytic solution, obtained in the weak-anisotropy approximation, provides a convenient way to estimate the impact of the distortions of the radiation patterns on AVO results. It is shown that the shape of the $\mathrm{P}$-wave radiation pattern in the range of angles most important to AVO analysis $\left(0-40^{\circ}\right)$ is mostly dependent on he difference between Thomsen parameters $\epsilon$ and $\delta$. For media with $\epsilon-\delta>0$ (the most common case), the P-wave amplitude may drop substantially over the first $25^{\circ}-40^{\circ}$ from vertical. There is no simple correlation between the strength of velocity anisotropy and angular amplitude variations: for instance, for models with a fixed positive $\epsilon-\delta$ the amplitude distortions are less pronounced for larger anisotropies $\epsilon$ and $\delta$. The distortions of the SV-wave radiation pattern are usually much more significant than those for the P-wave.

The anisotropic directivity factor for the incident wave may be of equal or greater importance for AVO than the influence of anisotropy on the reflection coefficient. Therefore, interpretation of AVO anomalies in the presence of anisotropy requires an integrated approach that takes into account not only the reflection coefficient but also the wave propagation above the reflector.
\end{abstract}




\section{INTRODUCTION}

Amplitude-versus-offset (AVO) analysis is one of the few exploration methods capable of direct detection of hydrocarbons. Conventional AVO algorithms are based on analytic expressions for $\mathrm{P}$-wave reflection coefficient valid for isotropic media. The presence of elastic anisotropy on either side of the reflector may significantly distort the angular dependence of reflection coefficients (e.g., Keith and Crampin, 1977; Banik, 1987; Wright, 1987). Banik (1987) and Thomsen (1993) developed analytic approximations for the reflection coefficient at a boundary between two transversely isotropic media in the limit of weak anisotropy and of small velocity and density contrasts across the reflector. A numerical analysis of the P-wave reflection coefficient at the interface between anisotropic shales and isotropic gas sands was given by Kim et al. (1993). Yet another substantial distortion of the amplitude-versus-offset (AVO) signature in anisotropic media is associated with the wave propagation above the reflector.

The real goal of AVO is to perform reflection coefficient-versus-angle analysis rather than study directly amplitude-versus-offset dependence. Hence, correction for the angular amplitude variation caused by the wave phenomena above the reflector is an essential component of AVO technology. Propagation effects, usually described in the ray approximation, include source directivity, energy divergence, and transmission and attenuation losses along the raypath (Duren, 1992). Although correction for propagation phenomena in realistic inhomogeneous subsurface models encounters many practical difficulties (Martinez, 1993), it is well understood if the medium is isotropic.

If the velocity above the reflector is angle-dependent, the behavior of body-wave amplitudes becomes much more complicated, and the isotropic correction becomes inadequate. The presence of anisotropic layers above the target horizon may be quite typical for sand-shale sequences commonly considered in AVO analysis (Kim et al., 1993). While reservoir sands can be expected to exhibit very weak anisotropy (if any), shale formations are often characterized by strong transverse isotropy, i.e., pronounced velocity variations in the incidence (vertical) plane (White et al., 1983; Robertson and Corrigan. 1983; Banik, 1984; Sams et al., 1993, among others).

Tsvankin and Chesnokov (1990a; hereafter referred to as TC90) gave a systematic description of point-source radiation patterns in azimuthally isotropic and orthorhombic media. Along with numerical analysis based on evaluation of Fourier-Bessel integrals, TC90 presented an analytic solution for radiation patterns derived in the stationary-phase approximation. Results of TC90 showed that the distribution of energy along wavefronts in anisotropic media is substantially distorted by focusing and defocusing of energy, usually associated with maxima and minima, respectively, in the angle-dependent velocity.

Asymptotic ray-theory expressions for radiation patterns in anisotropic media have been derived by Ben-Menahem et al. (1991) and Gajewski (1993). Gajewski 
(1993) developed an efficient numerical scheme to evaluate the Gaussian curvature of the slowness surface needed to calculate radiation patterns, and gave a detailed discussion of body-wave amplitudes in models containing aligned liquid-filled and dry cracks. A numerical example illustrating the influence of the distortion of $\mathrm{P}$-wave radiation patterns on AVO was presented by Samec and Blangy (1992).

The main goal of this paper is to study the influence of the distortions of bodywave radiation patterns in transversely isotropic media on amplitude-versus-offset analysis. The existing solutions for point-source radiation in anisotropic models require numerical evaluation and do not provide easy analytic insight into the problem. Here, I present a concise weak-anisotropy approximation for radiation patterns in transversely isotropic media that relates the distortions of point-source radiation to Thomsen parameters $\epsilon, \delta$ (for the P-and SV-wave), and $\gamma$ (for the SH-wave). The weak-anisotropy solution is compared with results of exact numerical modeling and with the stationary-phase approximation developed in TC90. Analysis of radiation patterns in the range of angles most important for AVO is followed by the comparison of the influence of transverse isotropy on two principal components of AVO signature - the wave propagation above the reflector and the reflection coefficients.

\section{GENERAL ANALYSIS FOR TRANSVERSE ISOTROPY}

Far-field point-source radiation in isotropic homogeneous, non-attenuating media is determined just by the source directivity factor and spherical divergence of amplitude (Aki and Richards, 1980). The far-field approximation for source radiation in anisotropic media, derived in TC90 by means of the stationary-phase method, is a much rnore complicated function that depends on the shape of the slowness surface. The most significant distortion of radiation patterns in anisotropic media is caused by the phenomena defined in TC90 as "focusing" and "defocusing" of energy. Energy increases (focuses) in parts of the wavefront with high concentration of groupvelocity vectors of elementary plane waves (which comprise point-source radiation). Conversely, defocusing corresponds to areas with low concentration of group-velocity vectors. Often (but not always), focusing takes place near velocity maxima, while defocusing is often associated with velocity minima.

Quantitative analysis of radiation patterns in TC 90 was performed by a numerical technique based on plane-wave decomposition of point-source radiation with subsequent evaluation of Fourier-Bessel integrals in the frequency domain. This numerical method involves an approximate treatment of azimuthal anisotropy; lowever, it is exact for azimuthally isotropic media. The stationary-phase approximation was used in TC90 only for qualitative estimates. Here, the stationary-phase solution for radiation patterns from TC90 is transformed into a much simpler expression valid for weak transverse isotropy. The accuracy of the weak-anisotropy formula is then checked by comparison with the original stationary-phase result as well as with the exact radiation patterns generated by evaluating Fourier-Bessel integrals.

I consider a simple model of a horizontal reflector below a transversely isotropic 
medium with a vertical symmetry axis (VTI) (Figure 1). Transverse isotropy will be described by the vertical velocities $V_{P 0}$ and $V_{S O}$ of $\mathrm{P}$ - and S-waves respectively (the qualifiers in "quasi-P-wave" and "quasi-SV-wave" will be omitted for brevity) and three dimensionless anisotropic parameters $\epsilon, \delta$ and $\gamma$ introduced by Thomsen (1986):

$$
\begin{gathered}
\epsilon \equiv \frac{c_{11}-c_{33}}{2 c_{33}}, \\
\delta \equiv \frac{\left(c_{13}+c_{44}\right)^{2}-\left(c_{33}-c_{44}\right)^{2}}{2 c_{33}\left(c_{33}-c_{44}\right)} \\
\gamma \equiv \frac{c_{66}-c_{44}}{2 c_{44}}
\end{gathered}
$$

where $c_{i j}$ are stiffness coefficients.

Although originally designed for weakly anisotropic models, the parameters $\epsilon, \delta$ and $\gamma$ are convenient to use in TI media with arbitrary strength of velocity anisotropy (Tsvankin and Thomsen, 1994; Tsvankin, 1994).

P-SV propagation is described by four coefficients $\left(V_{P 0}, V_{S 0}, \epsilon\right.$, and $\left.\delta\right)$, while the SH-wave velocity depends on $V_{S 0}$ and $\gamma$. Analytic developments for the SHwave in transversely isotropic media are relatively straightforward because SH-wave anisotropy is elliptical. At the same time, for $\mathrm{P}$ and SV-waves elliptical anisotropy is just a special case of transverse isotropy that occurs if $\epsilon=\delta$; existing data indicate that the elliptical condition is seldom satisfied by real rocks (Thomsen, 1986).

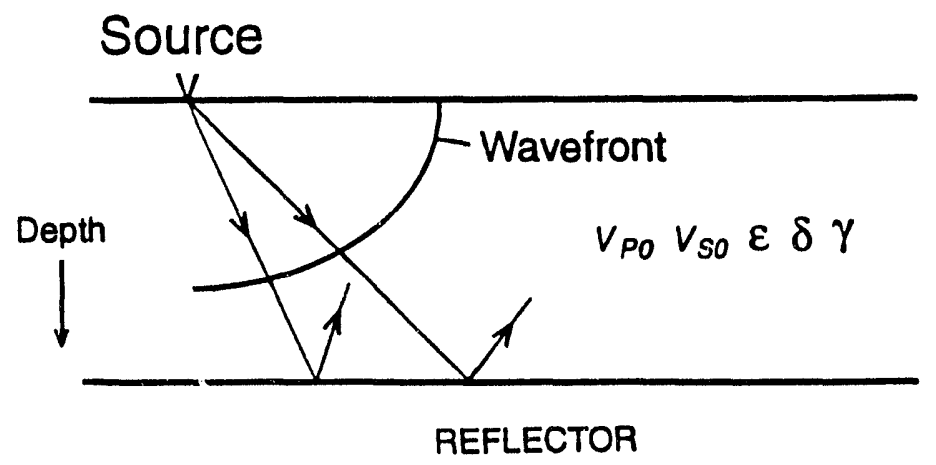

FIG. 1. Reflection from the bottom of a transversely isotropic layer. The redistribution of energy along the wavefront of the incident wave may distort the AVO response.

The influence of the free surface on radiation patterns is not taken into account, nor is that of source and receiver arrays; here I concentrate on the propagation phenomena caused by velocity anisotropy. These anisotropy-induced distortions of wavefront amplitudes are quite general since they take place not only in the source layer (we 
seldom observe anisotropy immediately below the surface) but in any other anisotropic layer encountered by the ray. The pure influence of the wavefront focusing phenomena is also of prime interest in cross-hole and reverse VSP surveys, which employ buried sources.

It is important to mention that the radiation patterns in this paper are derived as a function of the phase or group angle with the symmetry axis; therefore, the analytic developments below can be easily applied not only for VTI media, but also for transverse isotropy with any orientation of the axis of symmetry.

Derivation of the weak-anisotropy approximation for point-source radiation is given in the Appendix. The far-field radiation pattern of $\mathrm{P}, \mathrm{SV}$, or $\mathrm{SH}$-waves from a point force for weak transverse isotropy $(\epsilon \ll 1, \delta \ll 1, \gamma \ll 1)$ is shown to be

$$
U(R, \theta)=\frac{F_{u}}{4 \pi \rho V^{2}(\theta) R} \frac{1}{\sqrt{\frac{\sin \psi}{\sin \theta}\left(1+\frac{1}{V} \frac{d^{2} V}{d \theta^{2}}\right)}},
$$

where $U$ is the magnitude of the displacement, $\theta$ is the phase angle measured from vertical, $V$ is the phase velocity, $\rho$ is the density, and $R=\sqrt{z^{2}+r^{2}}(z$ is the receiver depth, $r$ is the horizontal source-receiver offset). The source term $F_{u}$ is the projection of the force on the displacement (polarization) vector. Expression (4) should be evaluated at the phase angle $\theta$, corresponding to a given ray (group-velocity) angle $\psi=\tan ^{-1}(r / z)$ of the incident wave; it can be shown that at velocity maxima or minima the phase and group velocity vectors coincide with each other.

Formula (4) clearly demonstrates how point-source radiation is distorted by velocity anisotropy. The term $F_{u} /\left(4 \pi \rho V^{2} R\right)$ formally coincides with the well-known expression for the far-field point-force radiation in isstropic media (Aki and Richards, $1980)$. However, the phase velocity in equation (4) is angle-dependent, so the term $V^{2}(\theta)$ reflects the direct influence of velocity variations on the radiation pattern. Since body-wave polarizations are dependent on the elastic constants (e.g., for the $\mathrm{P}$-wave the polarization vector deviates from the ray), the source term $F_{u}$ may also be distorted by the anisotropy. Also, the expression should be evaluated in the phase direction (angle $\theta$ ), which is generally different from the source-receiver direction due to the presence of anisotropy.

The term under the radical represents the pure contribution of the anisotropy to the radiation pattern. As shown in TC90, the second derivative of phase velocity is responsible for the focusing and defocusing phenomena mentioned above.

The distinction between the source term $F_{u}$ and the rest of formula (4) is very important. While $F_{u}$ is itself distorted by the anisotropy, the existence of the remaining anisotropic terms means that the redistribution of energy along the wavefront happens not only in the source layer, but also in any other anisotropic layer along the raypath.

In the absence of anisotropy equation (4) reduces to the expression for isotropic media given in Aki and Richards (1980). If the medium is not weakly anisotropic, 
formula (4) cannot be expected to be quantitatively accurate but may still be useful for qualitative estimates; a detailed analysis of the accuracy of the weak-anisotropy approximation is given below. Further linearization of equation (4) in the anisotropies $\epsilon, \delta$ and $\gamma$ leads to concise expressions for the P-, SV- and SH-waves that provide analytic insight into the amplitude distortions in transversely isotropic media.

\section{P-WAVE RADIATION PATTERNS}

\section{Weak-anisotropy approximation for $\mathrm{P}$-wave radiation}

The issue of $\mathrm{P}$-wave AVO is of particular importance because $\mathrm{P}$-waves constitute the overwhelming majority of all seismic data being used in exploration. The Pwave phase-velocity function for weak transverse isotropy $(\delta \ll 1, \epsilon \ll 1)$ is given by (Thomsen, 1986)

$$
V_{P}(\theta)=V_{P 0}\left(1+\delta \sin ^{2} \theta \cos ^{2} \theta+\epsilon \sin ^{4} \theta\right) .
$$

Equation (5) is fully linearized in the anisotropies $\epsilon$ and $\delta$. Differentiating (5) yields

$$
\begin{gathered}
\frac{d V_{P}(\theta)}{d \theta}=V_{P 0} \sin 2 \theta\left(\delta \cos 2 \theta+2 \epsilon \sin ^{2} \theta\right) \\
\frac{d V_{P}^{2}(\theta)}{d \theta^{2}}=2 V_{P 0}\left[\delta \cos 4 \theta+2 \epsilon \sin ^{2} \theta(1+2 \cos 2 \theta)\right]
\end{gathered}
$$

Also, for weak anisotropy

$$
\sin \psi=\sin \theta\left\{1+\cos ^{2} \theta\left[2 \delta+4(\epsilon-\delta) \sin ^{2} \theta\right]\right\}
$$

Substituting the above equations into formula (4) and further linearizing in $\delta$ and $\epsilon$, we obtain the weak-anisotropy approximation for the $\mathrm{P}$-wave radiation pattern,

$$
U_{P}(R, \theta)=\frac{F_{u}}{4 \pi \rho V_{P 0}^{2} R}\left[1-2 \delta-2(\epsilon-\delta) \sin ^{2} 2 \theta+\delta \sin ^{2} \theta\right] .
$$

From formula (9) it is clear that transverse isotropy distorts the amplitude even in the symmetry (vertical in our model) direction. In the weak-anisotropy equation (9), the anisotropic correction factor at $\theta=0$ reduces to $1-2 \delta$. For comparison, by using formula (A-4), it can be shown that the exact far-field $\mathrm{P}$-wave amplitude in the symmetry direction is given just by $F_{3} /\left[4 \pi \rho V_{P 0}^{2} R(1+2 \delta)\right]$, where $F_{3}$ is the vertical component of the force.

Therefore, the error of the weak-anisotropy approximation (9) in the symmetry direction is determined by the difference between the terms $1-2 \delta$ and $1 /(1+2 \delta)$. While this difference is small if $|\delta|<0.1$, for $\delta=0.2$ the amplitude error at normal incidence reaches 16 percent. More accurate absolute amplitudes in the weak-anisotropy 
approximation at small incidence angles can be obtained by skipping the last linearization step in equation (4) and retaining the anisotropy-related normal-incidence term in the form $1 /(1+2 \delta)$ :

$$
U_{P}(R, \theta)=\frac{F_{u}}{4 \pi \rho V_{P 0}^{2} R} \frac{1-2(\epsilon-\delta) \sin ^{2} 2 \theta+\delta \sin ^{2} \theta}{1+2 \delta} .
$$

Equation (10) yields the exact amplitude in the symmetry direction but, of course, may produce errors at oblique incidence if the anisotropies $\epsilon$ and $\delta$ are not small. In the following (including the numerical examples discussed in the next section), we will use equation (10) as the weak-anisotropy approximation for P-wave radiation.

The distortions of the $\mathrm{P}$-wave amplitude in the symmetry direction are directly related to the character of the velocity variations. Moreover, the focusing (or defocusing) of the $\mathrm{P}$-wave energy at vertical incidence depends on just one anisotropic coefficient $-\delta$, the parameter responsible for near-vertical $\mathrm{P}$-wave propagation. If $\delta<0$, the velocity function has a maximum at $\theta=0^{\circ}$, and the amplitude at vertical incidence increases due to the focusing of energy; conversely, if $\delta>0$, a velocity minimum leads to lower amplitudes at $\theta=0^{\circ}$ due to the defocusing. It should be emphasized that the velocity maximum or minimum in the symmetry direction represents the only "3-dimensional" velocity extremum in transversely isotropic media: phase velocity increases (or decreases) away from the symmetry axis in all directions, not just in the incidence plane. Therefore, the focusing (or defocusing) of energy in the symmetry direction is more pronounced than that for any other velocity extremum with the same value of $d V^{2} / d \theta^{2}$. This peculiarity of the symmetry direction is reflected in equation (4) by the value of the ratio $\sin \psi / \sin \theta$ at $\psi=\theta=0$ (equation [8]):

$$
\lim _{\theta \rightarrow 0} \frac{\sin \psi}{\sin \theta}=1+2 \delta,
$$

whereas $\sin \psi / \sin \theta=1$ at any other velocity extremum.

While distortions of absolute amplitude are certainly diagnostic of the presence of anisotropy, the property of radiation patterns of most importance in AVO analysis is the anisotropic correction to the angular amplitude distribution. Equation (10) shows that the lowest-order anisotropic angular correction to the radiation pattern near vertical is determined by the difference $\epsilon-\delta$. If $\epsilon-\delta>0$ (the most common case), transverse isotropy causes the $\mathrm{P}$-wave amplitude to decrease away from vertical. For elliptical anisotropy $(\epsilon=\delta)$, the term $2(\epsilon-\delta) \sin ^{2} 2 \theta$ vanishes, and the anisotropic angular correction reduces to $\delta \sin ^{2} \theta$. This means that for elliptical anisotropy $\mathrm{P}$-wave angular amplitude and velocity variations are in good correlation with each other because both depend similarly on the same parameter $-\delta$. However, the magnitude of the anisotropy-induced angular correction (given by $\delta \sin ^{2} \theta$ ) between $0^{\circ}$ and $40^{\circ}$ is relatively small, unless $\delta$ is unusually large. Finally, if $\epsilon-\delta<0$, we can expect an increase in the $\mathrm{P}$-wave amplitude with angle due to transverse isotropy. 
Angular distortions of the radiation pattern may also be caused by the source term $F_{u}$, which depends on the polarization vector. However, numerical results of TC90 and the analytic expressions discussed below prove that for moderate velocity anisotropy $\mathrm{P}$-wave polarization vector does not diverge much from the group (ray) direction. This means that source term $F_{u}$ for the P-wave is almost "isotropic", i.e., it is close to the absolute value of the cosine of the angle between the force and the group-velocity vector. For moderate anisotropies $|\epsilon| \leq 0.2,|\delta| \leq 0.2$, the distortions of the point-force directivity factor $F_{u}$ in the angular range $0-40^{\circ}$ are limited to a few percent. For more complex sources, such as dislocations or explosions, the dependence of the source term on anisotropy is more complicated, and can make a more significant contribution to the distortions of the angular amplitude distribution (i'svankin and Chesnokov, 1990b).

To explain the behavior of $\mathrm{P}$-wave polarizations and, consequently, of the term $F_{u}$, we have to find the polarization angle as a function of the anisotropic coefficients. In the weak-anisotropy approximation, this can be done by substituting the phase-velocity formula (5) into the Christoffel equations and obtaining the ratio of the vertical and horizontal displacement components. After carrying out further linearization in $\epsilon$ and $\delta$, we get

$$
\begin{gathered}
\tan \phi=\tan \theta\left\{1+B\left[2 \delta+4(\epsilon-\delta) \sin ^{2} \theta\right]\right\}, \\
B=\frac{1}{2\left(1-V_{S 0}^{2} / V_{P 0}^{2}\right)},
\end{gathered}
$$

where $\phi$ is the polarization angle measured from the symmetry axis.

I should note that in Thomsen's (1986) paper it is stated that the departure of the polarization angle from the phase angle for weak anisotropy is negligible. In fact, it can be shown that the P-wave polarization angle is closer to the group angle than to the phase angle. The tangent of the P-wave group angle (8) for weak anisotropy is given by

$$
\tan \psi=\tan \theta\left[1+2 \delta+4(\epsilon-\delta) \sin ^{2} \theta\right] .
$$

The expressions for the group (12) and polarization (11) angles are quite similar; the difference between the two is in the quantity $B$, which is dependent on the $V_{S 0} / V_{P 0}$ ratio. Since for plausible values of $V_{S 0} / V_{P 0}, B$ belongs to the interval $0.5<B<1$, the polarization vector always lies between the phase and group vectors, usually being closer to the group vector. Indeed, for a typical value of $V_{S 0}^{2} / V_{P 0}^{2}=1 / 3$, we get $B=0.75$, making the polarization and group vectors close to each other. This analytic result is in good agreement with the numerical analysis of $\mathrm{P}$-wave polarizations given in TC90.

It is more convenient to represent radiation patterns as a function of the group angle $\psi$ that determines the source-receiver direction $\left(\psi=\tan ^{-1}(r / z)\right)$. Formally, the 
difference between the phase and group angles in the anisotropic terms $2(\epsilon-\delta) \sin ^{2} 2 \theta$ and $\delta \sin ^{2} \theta$ can be simply ignored. If we replace $\theta$ in formula (10) by the group angle $\psi$ (using equation [8]) and do linearization in $\epsilon$ and $\delta$, we get exactly the same expression (10), but now with the group angle in place of the phase angle. Nevertheless, when the absolute values of the anisotropies approach the 15-20 percent range, the accuracy of the weak-anisotropy approximation is increased by evaluating expression (10) at the phase angle corresponding to a given group angle.

\section{Analysis of far-field P-wave amplitudes}

I begin by discussing the amplitude signature for the olivine model considered in TC90 and proceed with a systematic analysis for a suite of transversely isotropic models parametrized by $\epsilon$ and $\delta$. Figure 2 shows the $\mathrm{P}$-wave amplitudes excited by a point vertical force in the model of transversely isotropic olivine with $\epsilon-\delta=$ 0.051 . The dotted curve in Figure 2 is the exact result obtained by evaluating FourierBessel integrals as described in TC90; the solid curve is the stationary-phase solution, equation (A-4), valid in the far field for arbitrary strength of the anisotropy; and the dashed curve is the far-field weak-anisotropy approximation (10) (it can hardly be distinguished from the solid curve). The weak-anisotropy result was calculated using equations (8) and (11) for the group and polarization angles, respectively.

All three curves are normalized by the radiation pattern in the corresponding isotropic medium $(\epsilon=0, \delta=0)$, given by $\cos \psi /\left(4 \pi \rho V_{P_{0}}^{2} R\right)$ (for a unit force), to demonstrate the influence of the anisotropy on body-wave amplitudes. For an isotropic medium, the normalized amplitude curves would simply be equal to unity for all propagation angles. In addition to revealing the angular distortions, this correction makes it possible to see the difference in the absolute amplitude caused by the anisotropy.

For the olivine model in Figure 2, transverse isotropy causes the amplitude to decrease away from vertical up to a minimum near $45-50^{\circ}$. At a group angle of $45^{\circ}$, the exact normalized amplitude (dotted curve) is 14 percent lower than at vertical incidence. Note that the $\mathrm{P}$-wave phase velocity has a maximum at $\theta=0^{\circ}$ and a minimum near $\theta=49^{\circ}$. Thus, the decrease in the $\mathrm{P}$-wave amplitude is caused by the focusing of energy at vertical incidence and defocusing near $45-50^{\circ}$. Although a distortion of 14 percent over a $45^{\circ}$ interval does not seem to be very significant, it occurs in a medium with less than 2 percent percent maximum variation in the $\mathrm{P}$-wave phase velocity!

Since the radiation pattern in Figure 2 was evaluated in the far field, the solution obtained by the stationary-phase method (solid curve) should be close to the exact result (dotted curve). Indeed. the two curves in Figure 2 practically coincide with each other, which proves the accuracy of the asymptotic formula (A-4). The stationaryphase result diverges from the exact amplitude only in the vicinity of the minimum of the radiation pattern (located at $\psi=90^{\circ}$, not shown here) due to the nongeometrical effects described in TC90. The values of $\epsilon$ and $\delta$ for transversely isotropic olivine are 

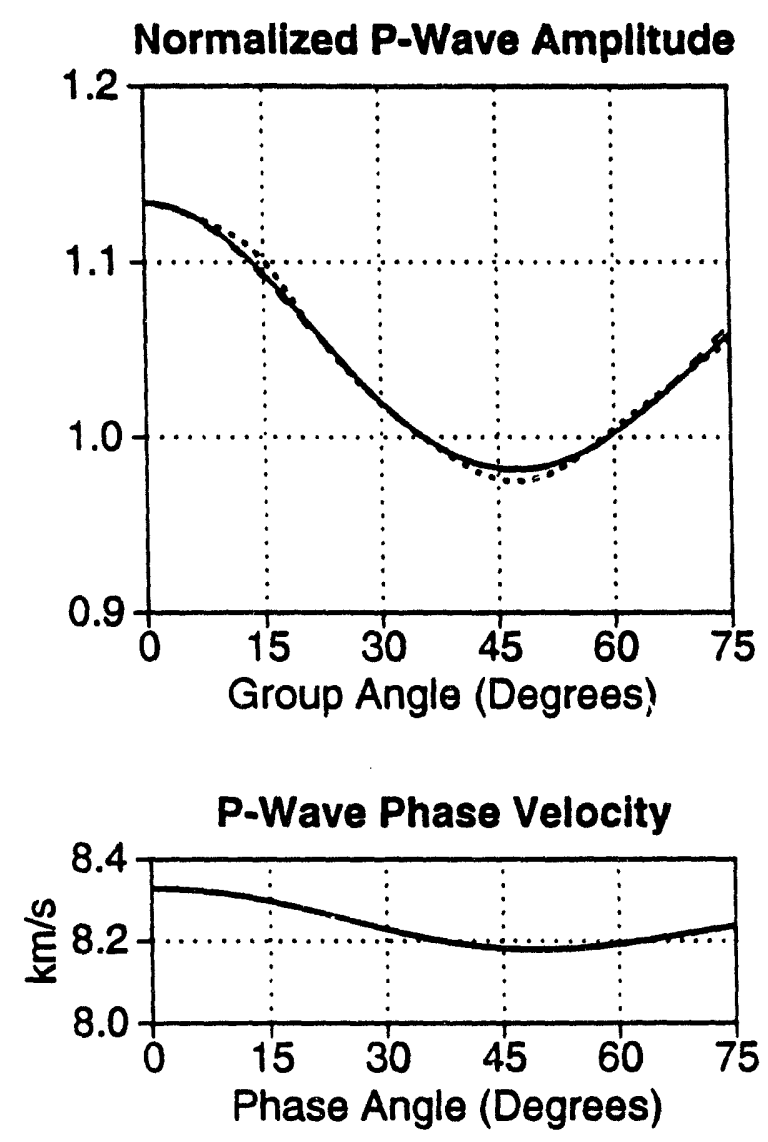

Fig. 2. P-wave amplitude from a point vertical force in transversely isotropic olivine. The dotted curve is the exact result obtained by evaluating Fourier-Bessel integrals; the solid curve is the stationary-phase solution (A-4); and the dashed curve is the weak-anisotropy approximation (10). All curves are normalized by the radiation pattern in the corresponding isotropic medium $(\epsilon=0, \delta=0)$.

The phase-velocity curve is shown at the bottom. The model parameters are: $V_{P 0}=8.328 \mathrm{~km} / \mathrm{s}, V_{S 0}=4.606 \mathrm{~km} / \mathrm{s}, \delta=-0.059, \epsilon=-0.008$. The receiver is located at the constant distance $R$ from the source in the far field $\left(R / \lambda_{p} \approx 12\right.$, where $\lambda_{p}$ is the average $P$-wavelength in the isotropy plane). 
small and, predictably, the accuracy of the weak-anisotropy approximation (dashed curve) is sufficiently high.

Before proceeding with the $\mathrm{P}$-wave amplitude analysis for a range of TI models, it is necessary to find out what parameters have an impact on $\mathrm{P}$-wave radiation. Clearly, the influence of transverse isotropy on $\mathrm{P}$-wave amplitudes is mostly determined by the anisotropies $\epsilon$ and $\delta$. However, $P$-wave radiation pattern also depends on the vertical velocities $V_{P O}$ and $V_{S O}$. While the P-wave velocity $V_{P O}$ is just a scaling coefficient that does not change the shape of the radiation pattern (for constant $\epsilon, \delta$, and $V_{P 0} / V_{S O}$ ), the contribution of the $\mathrm{S}$-wave velocity $V_{S O}$ to the $\mathrm{P}$-wave amplitude needs to be evaluated.

Tsvankin and Thomsen (1994) and Tsvankin (1994) showed that if a TI medium is parametrized by $V_{P 0}, V_{S 0}, \epsilon$ and $\delta$, the influence of the S-wave vertical velocity on $\mathrm{P}-\mathrm{r}_{f}$ ave phase and group velocities is practically negligible, even for strong anisotropy. The situation with the amplitudes looks more complicated. Although there is no explicit dependence on $V_{S 0}$ in the weak-anisotropy approximation (10), the source term $F_{u}$ is somewhat dependent on $V_{S 0}$ through the polarization direction [equation (11)]. The influence of $V_{S O}$ on the stationary-phase solution (A-4) might be expected to be stronger yet because several components of the Christoffel tensor in (A-4) contain the elastic coefficient $c_{44}$ and, consequently, the velocity $V_{S 0}$ (see Appendix).

However, as illustrated by Figure 3, not only the velocity but also the far-field amplitude of the $\mathrm{P}$-wave is practically independent of the S-wave vertical velocity. For $V_{P 0} / V_{S 0}$ ratio varying from 1.73 to 2.2 , the exact far-field amplitude in the 0 $40^{\circ}$ angular range changes by less than 1.5 percent. In the suite of plots starting with Figure 3, I show P-wave radiation patterns from a vertical force calculated using the stationary-phase expression (A-4) (solid curve), and the weak-anisotropy approximation (10) (dashed curve). As in Figure 2, both curves are normalized by the radiation pattern in the corresponding isotropic medium $(\epsilon=0, \delta=0)$. The exact phase velocity (solid curve) and its weak-anisotropy approximation (5) (dashed curve) are shown at the bottom.

Now, I continue with discussion of $\mathrm{P}$-wave amplitude signatures for a representative set of transversely isotropic models. The olivine model in Figure 2 represents a weakly anisotropic medium with small negative $\delta=-0.059$ and $\epsilon \approx 0$. While both positive and negative values of $\delta$ are plausible, $\epsilon$ is almost always positive (Thomsen, 1986). With decreasing $\delta$ and increasing $\epsilon(\epsilon>0, \delta<0)$, the second derivative of the velocity function ( 7 ) decreases more rapidly with angle. Consequently, the defocusing of energy away from vertical [see formula (4)] becomes more pronounced and spreads over a wider range of angles. It is important to mention that the maximum of energy defocusing in this case is shifted from the velocity minimum towards larger angles because $\frac{d V_{P}^{2}}{d \theta^{2}}$ continues to increase even beyond the velocity minimum. In formula (10), this defocusing trend manifests itself through the behavior of the two anisotropic angular terms.

Therefore, the example in Figure 2, corresponding to $\epsilon \approx 0$, illustrates minimum 


\section{$\varepsilon=0.25 \quad \delta=0.1$}
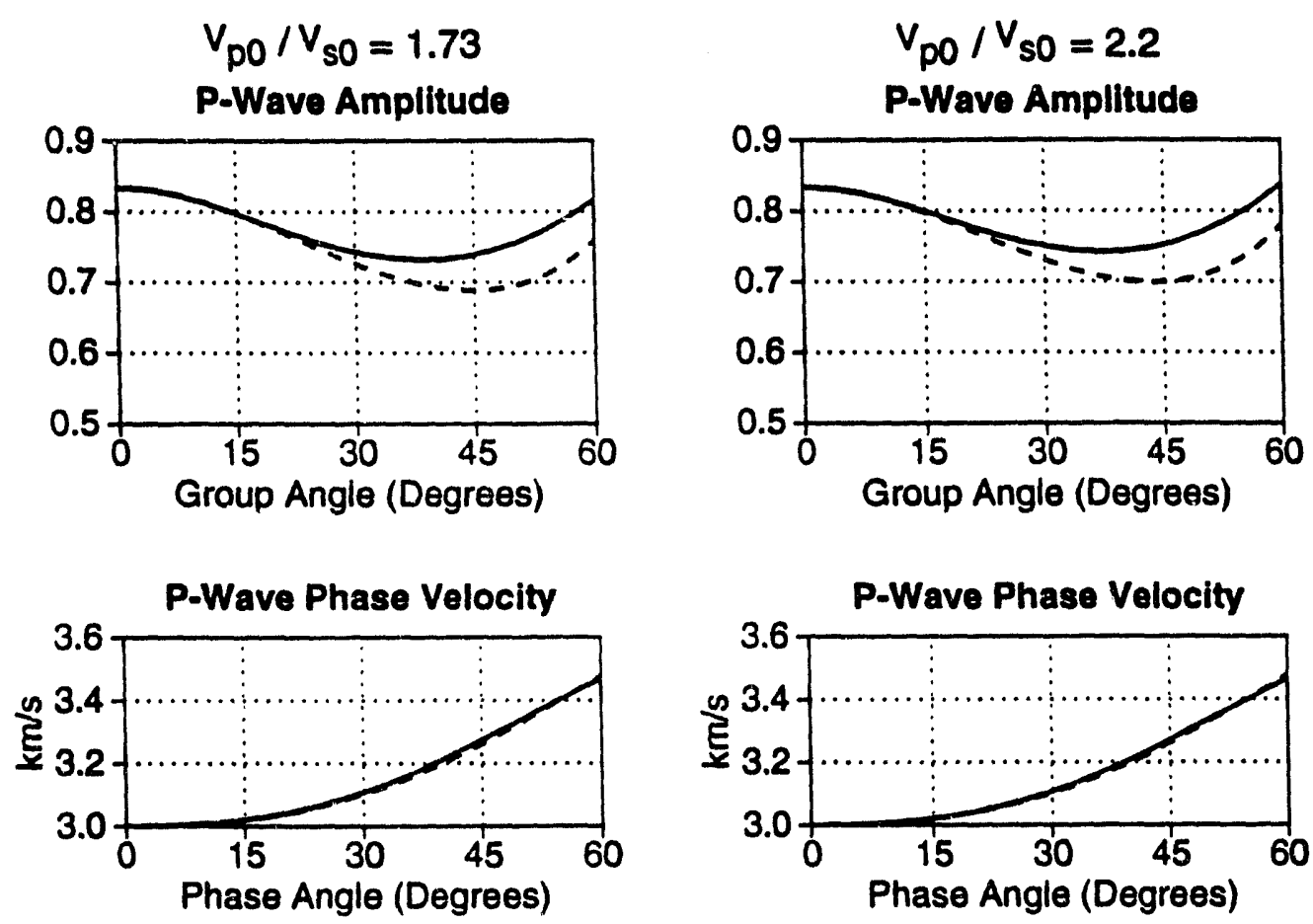

FIG. 3. The influence of the $\mathrm{S}$-wave vertical velocity on $\mathrm{P}$-wave amplitudes for the model with $\epsilon=0.25, \delta=0.1$, and $V_{P 0}=3 \mathrm{~km} / \mathrm{s}$. The solid curve is the stationaryphase solution (A-4); the dashed curve is the weak-anisotropy approximation (10). The amplitude curves are normalized by the radiation pattern in the corresponding isotropic model $(\epsilon=0, \delta=0)$. The plots at the bottom show the exact phase velocity (solid curve) and its weak-anisotropy approximation (5) (dashed curve).

expected distortions of the $\mathrm{P}$-wave radiation pattern for a small (plausible) negative value of $\delta$. For more typical weakly anisotropic models with $\delta<0, \epsilon>0(|\delta| \leq$ $0.1,|\epsilon| \leq 0.1)$, the $\mathrm{P}$-wave amplitude may drop by $20-30$ percent and more, from $0^{\circ}$ to $40^{\circ}$ (Figure 4; plot on the left).

From the results for $\delta<0$ (Figure 2 and left plot in Figure 4), one might get the impression that the $\mathrm{P}$-wave amplitude anomalies in the $0-40^{\circ}$ range resemble an amplified version of the shape of the phase-velocity function. However, this is not necessarily the case because, as indicated by the weak-anisotropy approximation, the shape of the P-wave radiation pattern is more controlled by the difference $\epsilon-\delta$ than by the individual values of the anisotropic coefficients. Two distinctly different phase-velocity functions in Figure 4 correspond to models with the same $\epsilon-\delta=0.1$ and, therefore, yield similar normalized amplitude curves with a decreasing trend away from vertical. Although there are no velocity minima between $0^{\circ}$ and $90^{\circ}$ for $\epsilon>\delta>0$, the second derivative of the phase-velocity function still increases with 


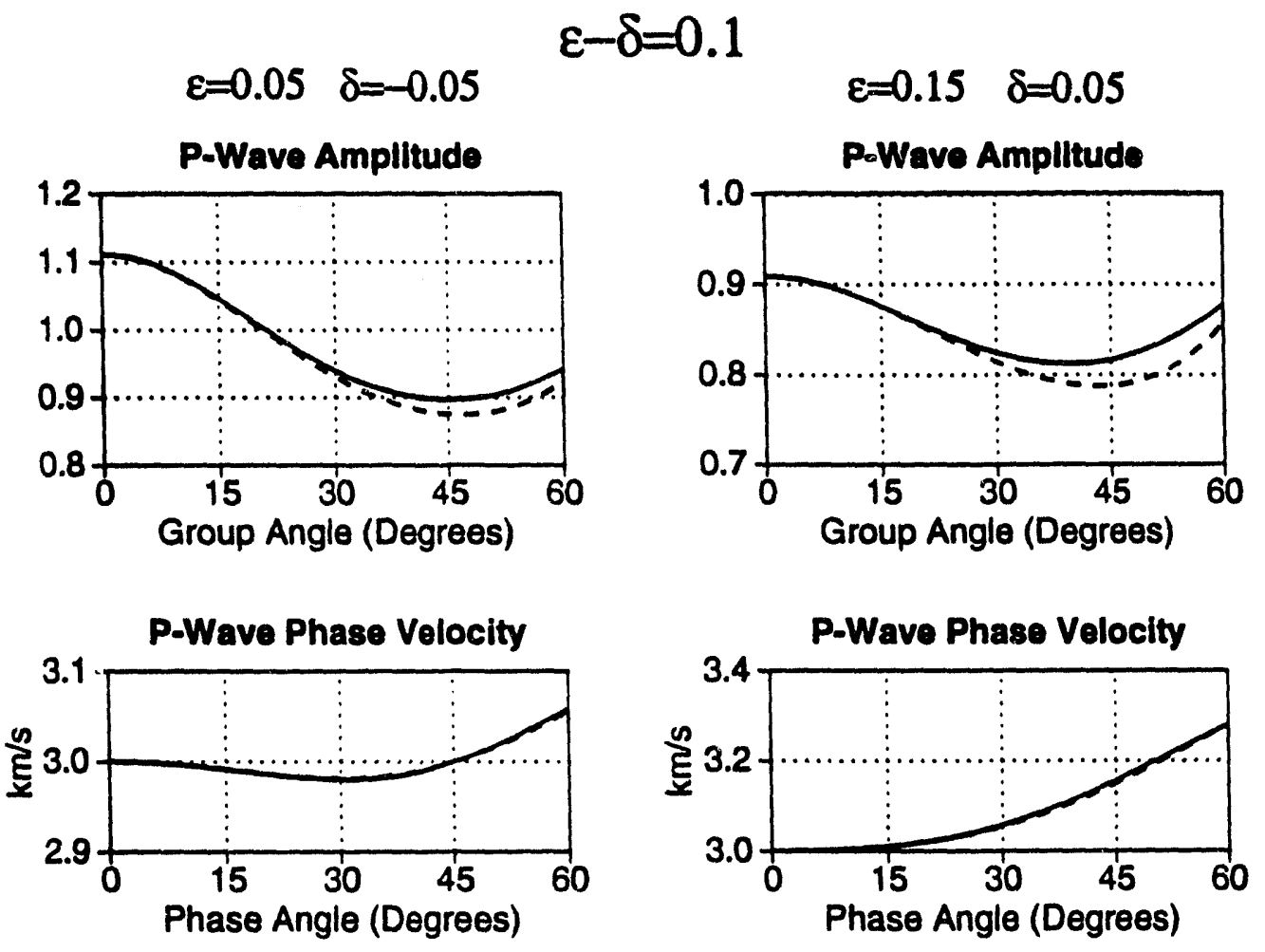

Fig. 4. P-wave amplitude generated by a vertical force for two models with the same $\epsilon-\delta=0.1$.

angle $\theta$. Since $V_{P}$ and $(\sin \psi / \sin \theta)$ in formula (4) also grow with angle, the P-wave amplitude decreases away from vertical (the right plot in Figure 4).

According to the existing measurements made at seismic frequencies, typically $\epsilon>\delta$ (Thomsen, 1986; Tsvankin and Thomsen, 1994). For instance, $\epsilon>\delta$ for transverse isotropy caused by thin bedding of isotropic layers (Berryman, 1979). Therefore, the decrease in the amplitude with angle may be typical for $\mathrm{P}$-wave propagation through transversely isotropic subsurface formations, such as shales.

In spite of the apparent similarity, there is a certain difference between the $\mathrm{P}$-wave amplitude signatures for the two models in Figure 4. First, the anisotropy leads to a higher amplitude at vertical incidence for the model with $\delta=-0.05$ and a lower amplitude for $\delta=0.05$ due to the focusing/defocusing phenomena near vertical, discussed above. Second, the angular amplitude variations (that are of primary interest to us in this problem) are more mild for the medium with larger values of $\epsilon$ and $\delta$. While for the model with $\epsilon=0.05$ the normalized amplitude drops by about 19 percent from $0^{\circ}$ to $40^{\circ}$, for the medium with $\epsilon=0.15$ the decrease in the same range is around 11 percent.

Although the weak-anisotropy formula (10) gives slightly lower amplitudes for angles beyond $30^{\circ}$ than the exact far-field result, the overall accuracy of the weak- 

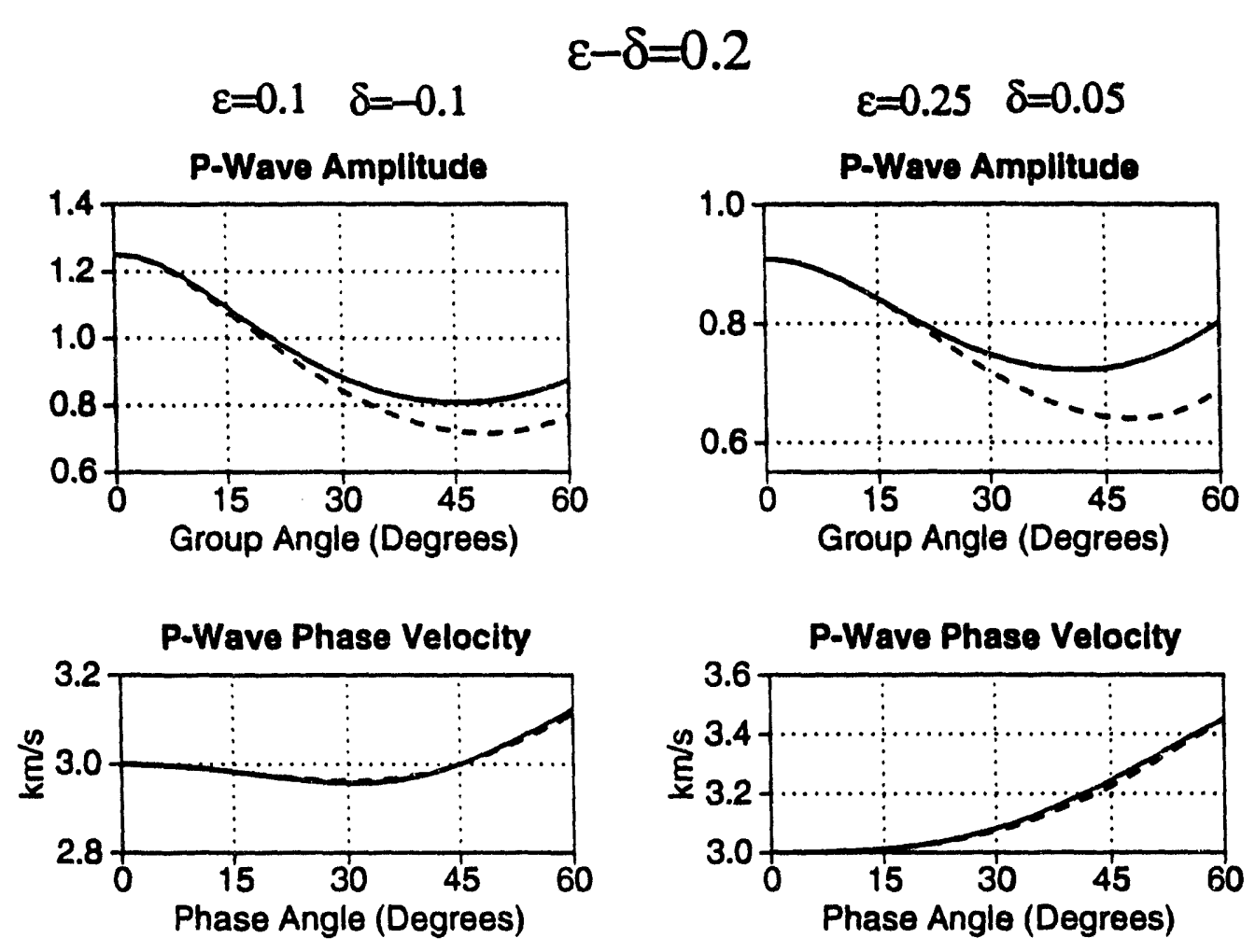

FIG. 5. Normalized P-wave amplitude from a vertical force for models with $\epsilon-\delta=0.2$.

anisotropy approximation for both models is quite satisfactory.

The angular dependence of $\mathrm{P}$-wave amplitudes for two media with a larger positive $\epsilon-\delta=0.2$ is shown in Figure 5 . As predicted by the weak-anisotropy approximation, the influence of the anisotropy on the $\mathrm{P}$-wave amplitude becomes more pronounced with increasing $\epsilon-\delta$. For the model on the left $(\epsilon=0.1, \delta=-0.1)$, which can be formally considered as weakly anisotropic, the drop in the normalized amplitude (using the solid curve) from $0^{\circ}$ to $40^{\circ}$ reaches 35 percent! For the model on the right $(\epsilon=0.25, \delta=0.05)$, despite a much larger value of $\epsilon$ and more significant phasevelocity variations, the amplitude distortions are more moderate (about 21 percent).

As illustrated by this example, the terms "weak anisotropy" or "strong anisotropy" are meaningless without a reference to a particular problem. While the model with $\epsilon=0.1, \delta=-0.1$ is weakly anisotropic in terms of velocity variations, it definitely qualifies as strongly anisotropic in terms of P-wave amplitude distortions. Different seismic phenomena (e.g., group velocities, normal moveout velocities, dip-moveout signatures, amplitudes etc.) depend on different combinations of anisotropic coefficients. These effective parameters are not easy to infer from the exact solutions. The power of the weak-anisotropy approximation is in providing a convenient tool for developing analytic insight into the influence of anisotropy on wave propagation. 
At the same time, weak-anisotropy solutions cannot be regarded as substitutes for the exact equations in the implementation of inversion and processing algorithms in anisotropic media.

In order to explain the difference between the amplitude signatures for the two models in Figure 5 in terms of the weak-anisotropy approximation (10), we have to take into account not only the explicit angular terms, but also the separation between the phase and group angles as well as the influence of the propagation direction on the source term $F_{u}$. Both factors are no longer negligible for the model with $\epsilon=0.25$, $\delta=0.05$, and both lead to an increase in the amplitude in the $30-40^{\circ}$ range. This, in turn, reduces the angular variation in the normalized amplitude.

While the weak-anisotropy approximation provides a valid qualitative description of the amplitude anomalies, it deviates from the exact solution with increasing angle. For the model with $\epsilon=0.25, \delta=0.05$, the exact amplitude at a group angle of $40^{\circ}$ is about 10 percent higher than the weak-anisotropy result. Although this error cannot be considered as large given the value of $\epsilon$, it may still look surprising because the weak-anisotropy phase-velocity curve in the $0-60^{\circ}$ range is close to the exact one (Figure 5). However, we should keep in mind that while deriving the weakanisotropy expression (10), we have linearized in $\epsilon$ and $\delta$ not only the phase velocity itself, but also its two derivatives, the group-velocity angle, the expression for $|\vec{U}|_{p t}$ containing the components of the Christoffel matrix (see the Appendix), and, finally, the fraction in equation (4). This multiple linearization may lead to much higher errors in the amplitude than those in the phase velocity function. For the model with $\epsilon=0.25, \delta=0.05$, the weak-anisotropy formula overstates the second derivative of phase velocity for incidence angles $30-50^{\circ}$ (as can be seen in the velocity plot in Figure 5) and, consequently, understates the amplitude in this angular range.

For elliptical anisotropy $(\epsilon=\delta$, Figure 6$)$, the stationary-phase solution reduces to a simple function of the group angle $\psi$ without application of the weak-anisotropy approximation:

$$
U_{P}(\text { elliptical })=\frac{F_{u}}{4 \pi \rho V_{P 0}^{2} R} \frac{1}{\sqrt{(1+2 \delta)\left(1+2 \delta \cos ^{2} \psi\right)}} .
$$

In elliptically-anisotropic models, the influence of anisotropy on $\mathrm{P}$-wave radiation pattern is well-correlated with the character of the velocity variations. If the value of $\epsilon=\delta$ is positive, both the velocity and the normalized amplitude increase away from vertical but the amplitude variations in the angular range $0-40^{\circ}$ remain mild, even for models with significant velocity anisotropy (Figure 6).

The similarity in the angular amplitude variation for elliptically anisotropic and isotropic models is easy to infer from the weak-anisotropy expression (10), as discussed above. For the medium with $\epsilon=\delta=0.25$, the exact far-field normalized amplitude (solid curve) increases just by 13 percent from $0^{\circ}$ to $40^{\circ}$. This amplitude distortion is partly due to the source term $F_{u}$ because the $\mathrm{P}$-wave polarization vector deviates 


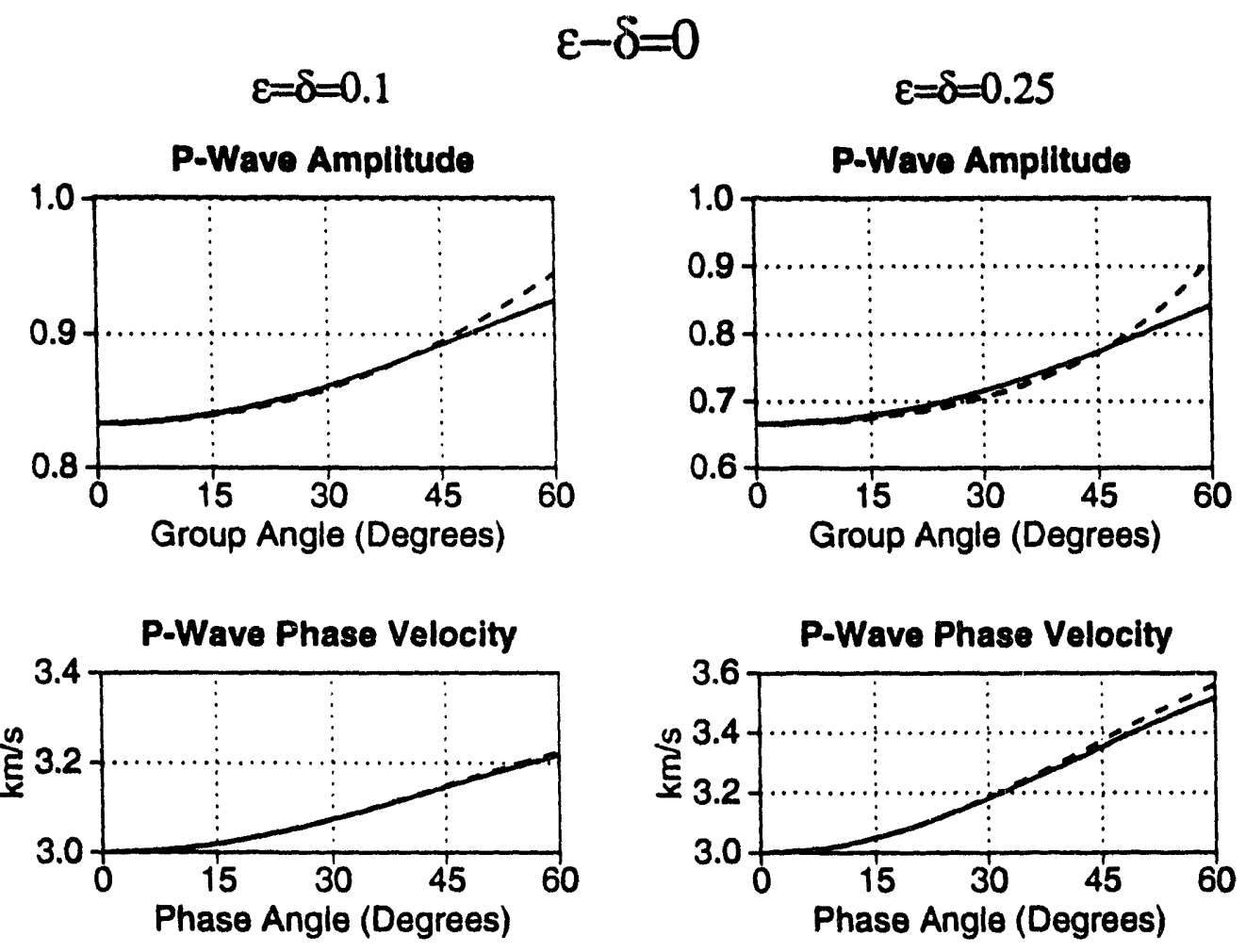

Fig. 6. Normalized P-wave amplitude from a vertical force for models with $\epsilon-\delta=0$ (elliptical anisotropy).

from the source-receiver (group) direction towards lower velocities, i.e., moves closer to the force vector. Elliptical anisotropy, however, may lead to a more significant change in the absolute value of the amplitude: for the same model with $\epsilon=\delta=0.25$, for example, the amplitudes near vertical are about 30 percent smaller than in the isotropic medium with the same $V_{P 0}$ and $\rho$.

To complete the analysis of $\mathrm{P}$-wave radiation patterns, I next examine amplitude signatures for models with negative $\epsilon-\delta$ (Figure 7 ). In this (less typical) case, in agreement with the weak-anisotropy result (10), the anisotropy leads to an increase in the P-wave amplitude with angle. Note that for models with $\epsilon-\delta<0$ the amplitude signature is much more controlled by the difference $\epsilon-\delta$ than for models with positive $\epsilon-\delta$. For both media in Figure $\bar{\tau}$, which have the same $\epsilon-\delta=-0.1$ but different individual values of $\delta$ and $\epsilon$, the increase in the normalized amplitude from $0^{\circ}$ to $40^{\circ}$ is almost the same $(\approx 25$ percent $)$.

The accuracy of the weak-anisotropy approximation turns out to be higher for elliptical anisotropy $(\epsilon=\delta)$ and models with negative $\epsilon-\delta$ than for media with $\epsilon-\delta>0$. Even for $\epsilon$ and $\delta$ in the $20-25$ percent range, the weak-anisotropy result does not deviate from the exact amplitude for incidence angles up to $40-45^{\circ}$. 


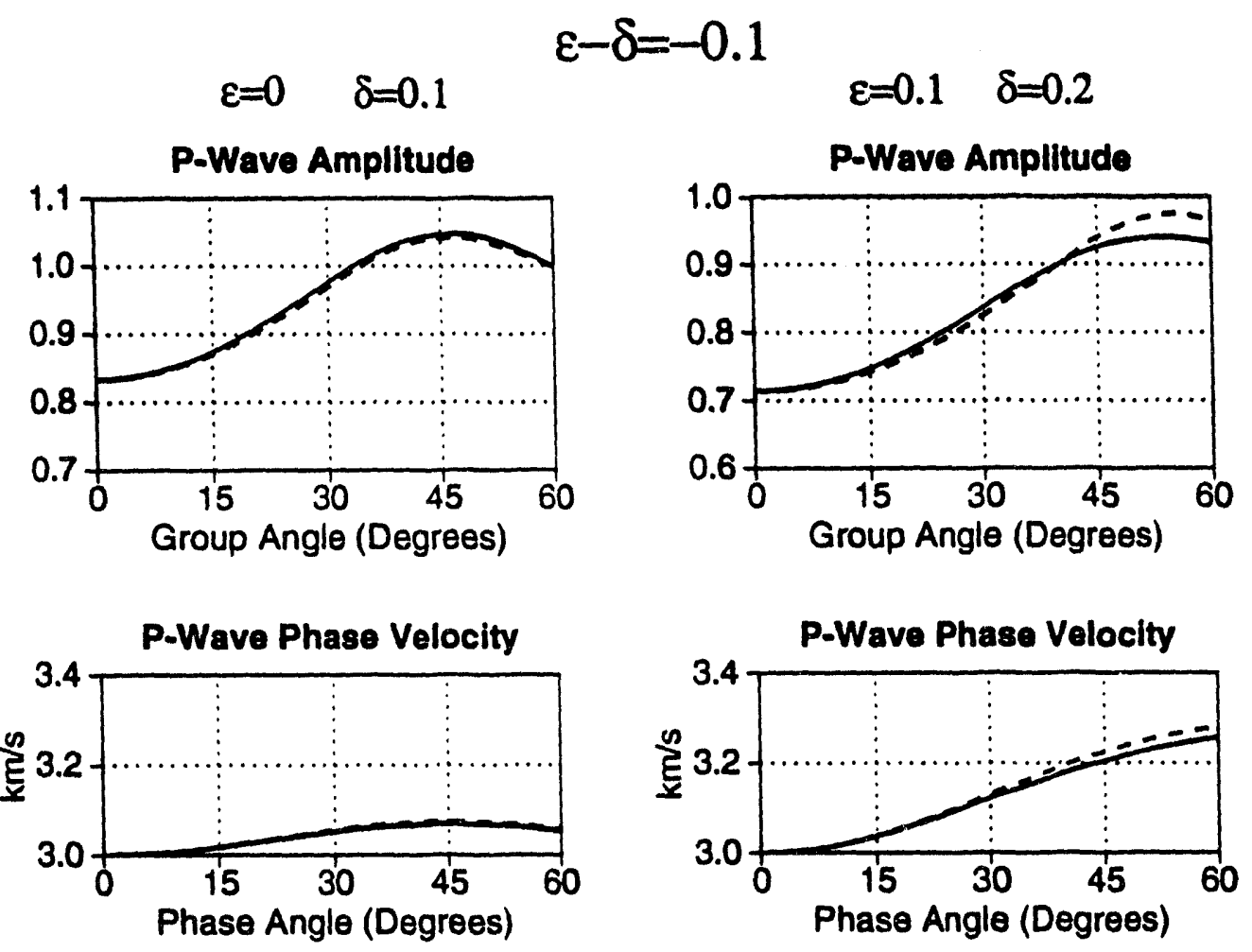

FIG. 7. Normalized P-wave amplitude from a vertical force for models with

$$
\epsilon-\delta=-0.1 \text {. }
$$

\section{Radiation pattern versus reflection coefficient}

How do the distortions of the $\mathrm{P}$-wave radiation pattern compare with the influence of transverse isotropy on the reflection coefficient? Thomsen (1993) gives the following approximation for the $\mathrm{P}$-wave reflection coefficient in the limit of weak transverse isotropy, and small velocity and density contrasts at the reflector:

$$
R(\theta)=R_{\text {isot }}(\theta)+R_{\text {anis }}(\theta),
$$

where $R_{\text {isot }}(\theta)$ is the reflection coefficient in the absence of anisotropy $(\epsilon=0, \delta=0)$, and

$$
R_{\text {anis }}(\theta)=\frac{1}{2}\left(\delta_{2}-\delta_{1}\right) \sin ^{2} \theta+\frac{1}{2}\left(\delta_{1}-\delta_{2}+\epsilon_{2}-\epsilon_{1}\right) \sin ^{2} \theta \tan ^{2} \theta
$$

Subscripts 1 and 2 refer to the media above and below the reflector respectively. One of the convenient features of equations (14) and (15) is the separation of the "isotropic" and "anisotropic" parts of the reflection coefficient. Formula (15) is an improvement over Banik's (1987) approximation, which is limited to small incidence angles. 
$\varepsilon=0.1 \quad \delta=-0.1$
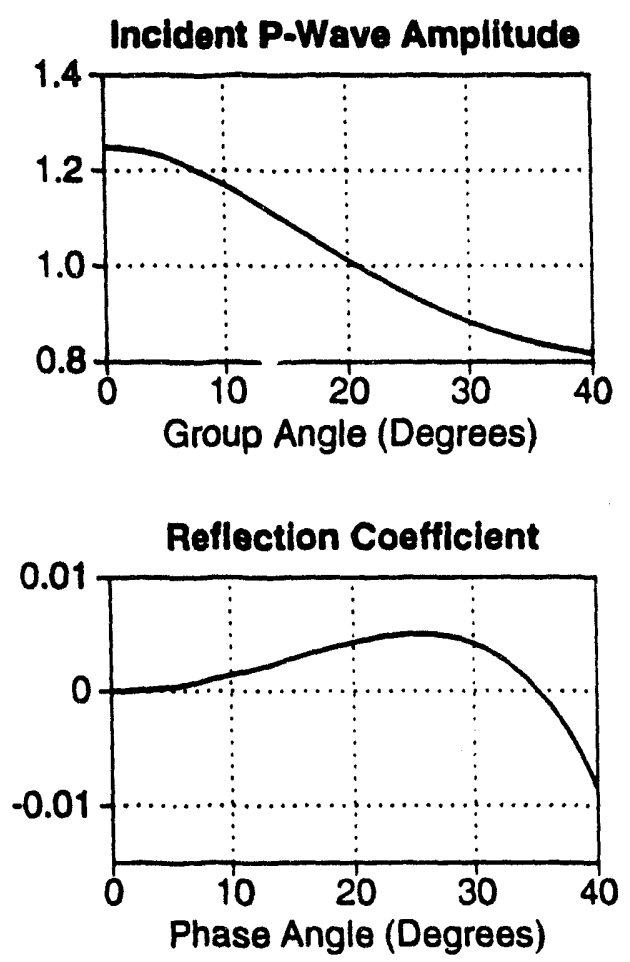

$\varepsilon=0.2 \quad \delta=0.1$
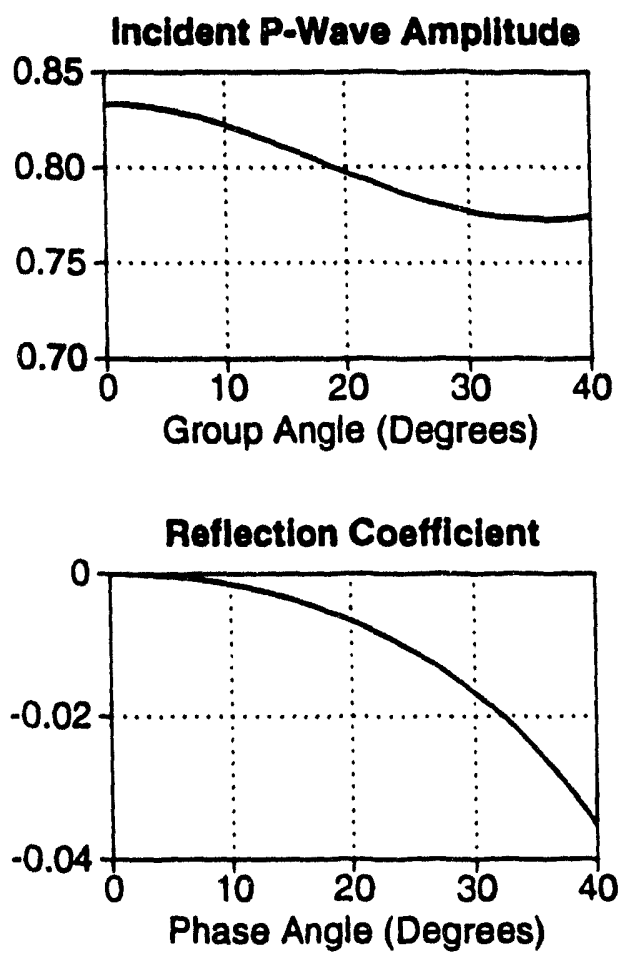

Fig. 8. Comparison of the influence of transverse isotropy on the P-wave radiation pattern from a vertical force and on the reflection coefficient.

(Top) The exact far-field amplitude of the incident wave from equation (A-4) normalized by the amplitude in the corresponding isotropic medium with $\epsilon=\delta=0$. (Bottom) The angular variation in the reflection coefficient caused solely by the anisotropy [equation (16)]. It is assumed that the transversely isotropic models with $\epsilon$ and $\delta$ shown on the plot overlie an isotropic medium.

Unlike the radiation pattern, the reflection coefficient at normal incidence is not distorted by transverse isotropy, if the density and $\mathrm{P}$-wave vertical velocity are the same in the isotropic and TI models. Note that the lowest-order angular correction to the reflection coefficient depends just on the change in $\delta$ across the reflector (no dependence on $\epsilon$ ), while the lowest-order angular term in the radiation pattern (10) contains the difference between $\epsilon$ and $\delta$.

To compare the influence of transverse isotropy on the radiation pattern and on the reflection coefficient, we assume that the medium below the reflector is isotropic (e.g., the shale/sand AVO model discussed by Kim et al., 1993). Then the anisotropic part of the reflection coefficent is determined just by $\epsilon$ and $\delta$ of the overlying medium:

$$
R_{\text {anis }}(\theta)=-\frac{1}{2} \delta_{1} \sin ^{2} \theta+\frac{1}{2}\left(\delta_{1}-\epsilon_{1}\right) \sin ^{2} \theta \tan ^{2} \theta
$$


For the two models shown in Figure 8, the importance of the radiation phenomena is quite different. If $\epsilon=0.1$ and $\delta=-0.1$, the anisotropy causes a 35 percent drop in the amplitude from $0^{\circ}$ to $40^{\circ}$, while the anisotropy-induced angular variations in the reflection coefficient do not exceed \pm 0.008 . Hence, for a typical value of the isotropic part of the reflection coefficient of about 0.1 , transverse isotropy would make less than a 10 percent change in the total reflection coefficient. This means that in this case the redistribution of energy above the reflector is likely to be a more important factor in AVO than the influence of the anisotropy on the reflection coefficient.

Conversely, for the model with $\epsilon=0.2$ and $\delta=0.1$, the distortions of the radiation pattern in the $0^{\circ}-40^{\circ}$ range are limited to 7 percent as compared with a change in the reflection coefficient of about 0.035 . Even if the reflection corresponds to a "bright spot" with a large absolute value of $R_{\text {isot }}$, we can expect the distortions of the reflection coefficient to be the dominant effect of transverse isotropy on AVO for this model.

Obviously, it is difficult to make a general comparison between the influence of anisotropy on the propagation phenomena and the reflection coefficient. The angular variations in the reflection coefficient depend on the difference in the anisotropic parameters across the reflector, while the radiation pattern is entirely determined by the properties of the incidence medium. Also, the influence of anisotropy on the reflection coefficient depends on the impedance contrast, i.e., it is more pronounced for weak reflectors. However, it is clear that the two phenomena are often of the same order of magnitude.

More examples of $\mathrm{P}$-wave reflection coefficients in transversely isotropic media are provided by Kim et al. (1993), who considered a shale/sand boundary under the assumption that only the shale (the medium above the reflector) is anisotropic. For moderate values of the anisotropies $\epsilon$ and $\delta$ in the shale, the difference between the isotropic and anisotropic reflection coefficients at an incidence angle of $40^{\circ}$ is usually limited by \pm 0.05 . In one of the typical cases considered by Kim et al. (1993) (Class 2 gas sands), this difference amounts to $30-35$ percent error in the reflection coefficient, which is comparable to the distortions of the radiation pattern we have discussed above.

In isotropic AVO analysis, the presence of gas is often identified by an increase in the absolute value of the $\mathrm{P}$-wave reflection coefficient with angle. Kim et al. (1993) concluded that transverse isotropy above the reflector usually enhances this behavior by further increasing the absolute value of the reflection coefficient away from vertical. Our analysis shows that the propagation phenomena in typical TI media (with $\epsilon-$ $\delta>0$ ) above the reflector may lead to a decrease in the amplitude with angle and, therefore, reduce or reverse the influence of the anisotropy on the reflection coefficient. For "bright spots" with a large amplitude of the normal-incidence reflection and a relatively slow increase in the absolute value of the reflection coefficient with angle (Class 3 sands from Kim et al., 1993), amplitude distortions above the reflector may even reverse the sign of the AVO gradient! 


\section{SHEAR-WAVE RADIATION PATTERNS}

\section{SV-wave}

The phase velocity of the SV-wave in transversely isotropic media is mostly determined by the effective parameter that Tsvankin and Thomsen (1994) denoted as $\sigma:$

$$
\sigma=\frac{V_{P 0}^{2}}{V_{S 0}^{2}}(\epsilon-\delta)
$$

In the weak-anisotropy approximation, the SV-wave phase velocity is given by

$$
V_{S V}(\theta)=V_{S 0}\left(1+\sigma \sin ^{2} \theta \cos ^{2} \theta\right) .
$$

The terms "weak" or "strong" velocity anisotropy for the SV-wave mostly refer to the value of $\sigma(17)$ rather than to the individual values of the anisotropies $\epsilon$ and $\delta$, although, of course, $\epsilon, \delta$, and $V_{P 0} / V_{S 0}$ ratio do have some separate influence on the $\mathrm{SV}$-wave velocity if $\sigma$ is not small.

Note that formula (18) can be obtained from the P-wave phase velocity [equation (5)] by replacing $V_{P 0}$ with $V_{S 0}, \delta$ with $\sigma$ and setting $\epsilon=0$. Since the radiation pattern in the weak-anisotropy approximation (4) is a function of phase velocity (except for the source term $F_{u}$ ), the SV-wave radiation pattern can be easily derived from $\mathrm{P}$-wave formula (10) by making the same substitutions $\left(V_{P 0}=V_{S 0}, \delta=\sigma, \epsilon=0\right)$ :

$$
U_{S V}(R, \theta)=\frac{F_{u}}{4 \pi \rho V_{S 0}^{2} R} \frac{1+2 \sigma \sin ^{2} 2 \theta+\sigma \sin ^{2} \theta}{1+2 \sigma} .
$$

In addition to maxima or minima at $\theta=0^{\circ}$ and $\theta=90^{\circ}$, the SV-wave phase velocity function has an extremum near $45^{\circ}$ (unless $\epsilon=\delta$, and $V_{S V}$ is constant). In the most common case of positive $\sigma(\epsilon>\delta)$, the SV-wave phase velocity has a minimum at vertical incidence followed by a maximum near $45^{\circ}$ (exactly as the P-wave when $\epsilon=0, \delta>0$ ). As shown by formula (19) and the analysis in the previous section, this leads to an increase in the incident wave amplitude with angle. An important difference. however, is that $\sigma$ is often much bigger than $\delta$ due to the contribution of the squared velocity ratio.

It is also noteworthy that the polarization direction and, consequently, the term $F_{u}$ is often much more distorted by transverse isotropy for the SV-wave than for the Pwave. Since the $\mathrm{P}$ and SV polarization vectors corresponding to the same phase angle are orthogonal, we can use the $\mathrm{P}$-wave formula (11) and just add $90^{\circ}$ to calculate the $\mathrm{SV}$ polarization direction. The group angle for the SV-wave in the weak-anisotropy approximation is given by (Thomsen, 1986)

$$
\tan \psi=\tan \theta(1+2 \sigma \cos 2 \theta)
$$


As follows from formulas (11) and (20), there is no simple correlation between the polarization and group angles for the SV-wave. This means that we can expect a more significant influence of transverse isotropy on $F_{u}$ for the $S V$-wave than for the P-wave.

Figure 9 shows the SV-wave amplitude for the same olivine model as in Figure 2, which has a positive value of $\epsilon-\delta$, thus yielding $\sigma>0$. The SV-wave velocity anisotropy (i.e., the maximum variation in velocity) for this model is only about 4.3 percent. Nevertheless, the focusing of energy near the velocity maximum causes a pronounced increase in the normalized SV-wave amplitude near the $45^{\circ}$ angle.
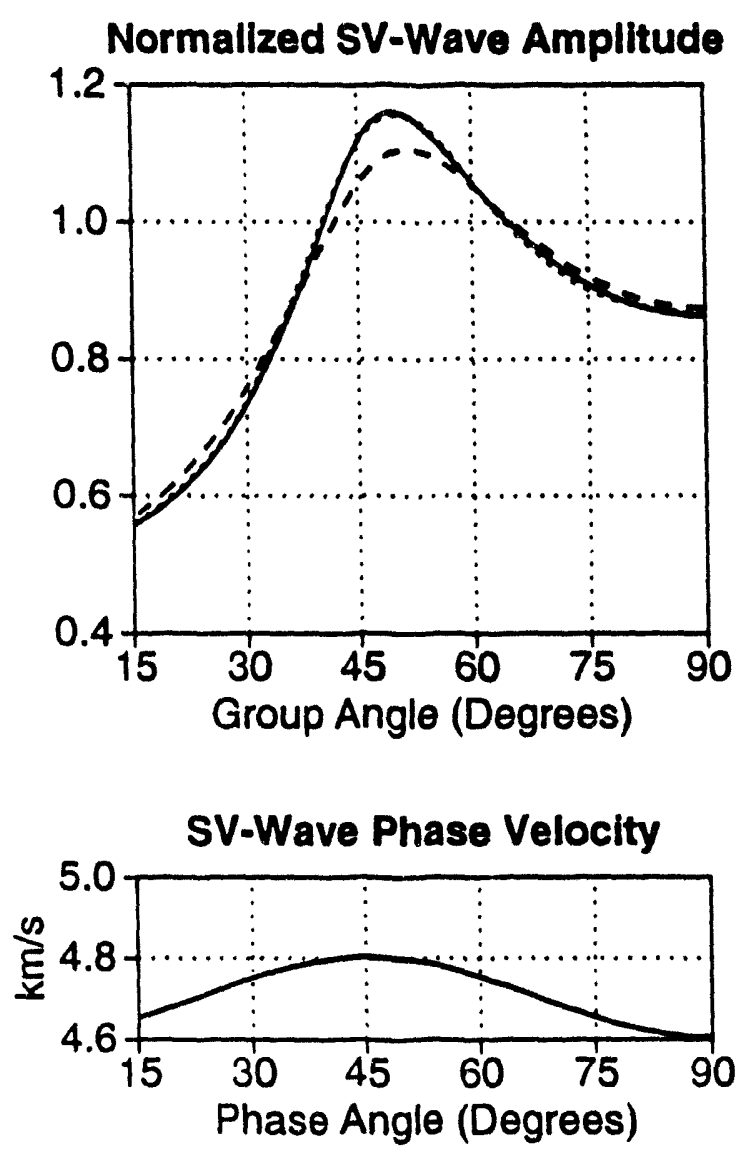

FIG. 9. SV-wave amplitude from a vertical point force in transversely isotropic olivine (model from Figure $2, \epsilon-\delta=0.051, \sigma=0.168$ ). The dotted curve is the exact result obtained by evaluating Fourier-Bessel integrals; the solid curve is the stationaryphase solution (A-4); and the dashed curve is the weak-anisotropy approximation (19) calculated using formulas (11) and (20). All three curves are normalized by the radiation pattern in the corresponding isotropic medium $(\epsilon=0, \delta=0)$.

The stationary-phase solution (solid curve) remains close to the exact amplitude (dotted curve) within the whole angular range shown in Figure 9 (the area near ver- 
$\sigma=0 \quad(\varepsilon=\delta=0.2)$

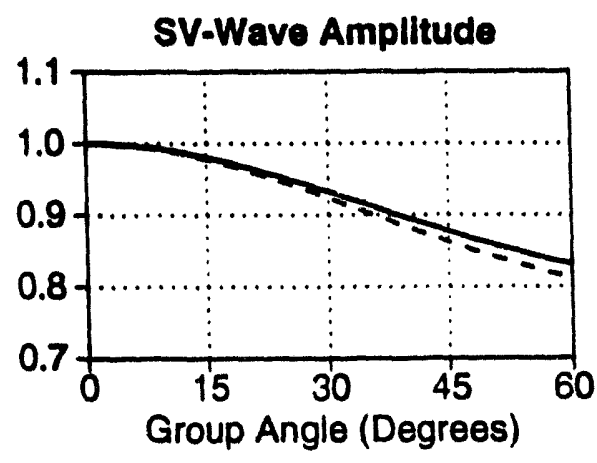

SV-Wave Phase Velocity

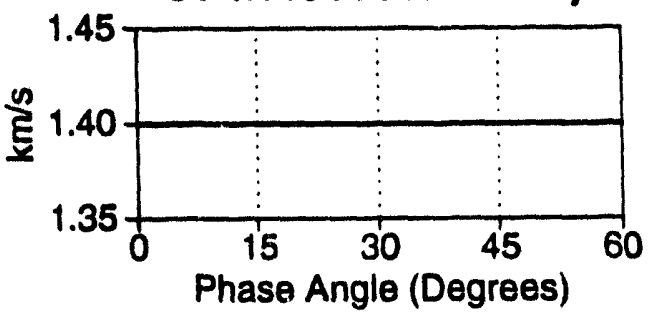

$\sigma=0.15 \quad(\varepsilon=0.1, \delta=0.05)$

SV-Wave Amplitude
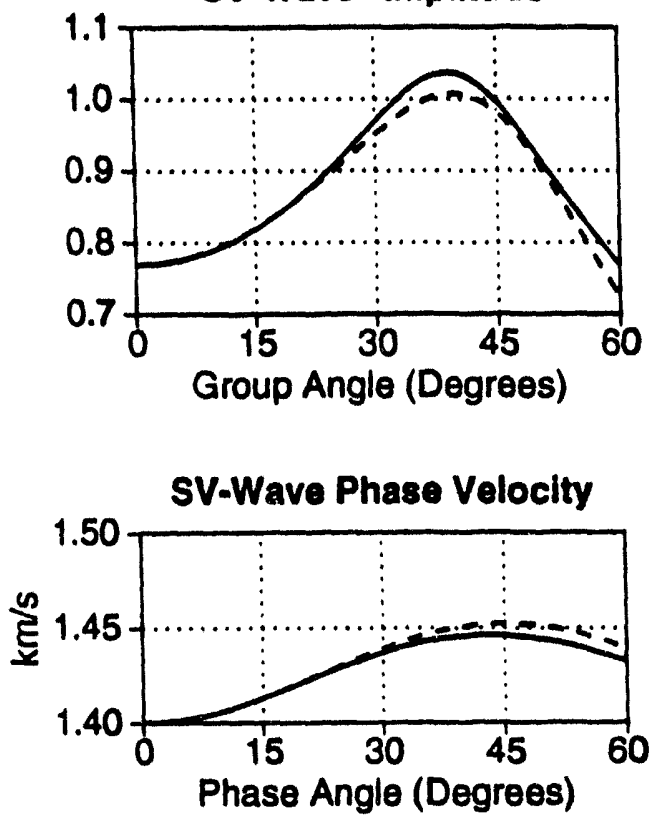

FIG. 10. SV-wave amplitude from a horizontal force for two VTI models with $V_{S 0}=1.4$ $\mathrm{km} / \mathrm{s}$; the anisotropic parameters are shown on the plot. The solid curve is the stationary-phase solution (A-4); the dashed curve is the weak-anisotropy approximation (19). The amplitude curves are normalized by the radiation pattern in the corresponding isotropic model $(\epsilon=0, \delta=0)$. The plots at the bottom show the exact phase velocity (solid curve) and its weak-anisotropy approximation (18) (dashed curve).

tical corresponds to the minimum of the radiation pattern where the amplitude goes to zero). This verifies the accuracy of the stationary-phase result (A-4) in describius far-field SV amplitudes. Although the coefficient $\sigma$ is not small $(\sigma=0.168)$, the error of the weak anisotropy approximation (19) for this model does not exceed 6 percent.

Figures 10 and 11 show normalized SV-wave radiation patterns from a horizontal force for a suite of models with $\sigma$ varying from zero (elliptical anisotropy) to 0.45 . The amplitudes were calculated using the stationary-phase solution (A-4) (solid curve) and the weak-anisotropy approximation (19) (dashed curve).

Elliptical anisotropy $(\sigma=0$, left plot in Figure 10) seems to be equivalent to isotropy for the SV-wave because the SV-wave phase and group velocities do not change with angle. However, even in this case the SV radiation pattern deviates from the isotropic one due to the influence of the source term $F_{u}$, i.e., due to the polarization anomalies. In the example with $\epsilon=\delta=0.2$ shown in Figure 10, the anisotropy tilts the SV-wave polarization vector towards vertical, thus reducing the amplitude generated by a horizontal force. It is interesting that for the SV-wave from 
a vertical force in the same model, the influence of the anisotropy leads to higher amplitudes. This explains the distortions of the SV-wave radiation pattern found by Ben-Menahem et al. (1991) and Gajewski (1993) for the elliptically anisotropic model of "Wills Point shale," which has $\epsilon=\delta=0.37$. It should be mentioned that the original elastic constants of Wills Point shale, experimentally determined by Robertson and Corrigan (1983), did not yield elliptical anisotropy; Ben-Menahem et al. (1991) adjusted the elastic coefficients reported by Robertson and Corrigan (1983) to make the model elliptical. On the whole, for elliptical anisotropy the SVwave amplitude distortions in the range $0-40^{\circ}$ are mild, unless the value of $\epsilon=\delta$ is unusually large.

Although the medium with $\epsilon-\delta=0.05$ (the right plot in Figure 10) seems to be close to elliptical, and the maximum velocity variation is just about 3.5 percent, the SV-wave amplitude signature is distinctly different from the one for elliptical anisotropy. The value of $\sigma=0.15$ is sufficient to make the normalized amplitude increase by 34.5 percent from $0^{\circ}$ to $40^{\circ}$.

For larger $\sigma$, the velocity minimum at vertical and the maximum near $45^{\circ}$ become sharper making the SV-wave amplitude anomaly much more pronounced (Figure 11). For the medium with $\sigma=0.45$, the increase in the normalized amplitude between $0^{\circ}$ and $40^{\circ}$ reaches an astonishing value of 170 percent, while the maximum SVwave velocity variation is only about 9 percent. These results are in good agreement with SV-wave focusing phenomena described in TC90 and Gajewski (1993). Here, however, the numerical results are supported by a consistent analytic treatment of the amplitude anomalies in terms of $\sigma$.

With a further increase in $\sigma$, the Gaussian curvature of the slowness surface at the velocity maximum decreases, eventually leading to a parabolic point, where the curvature goes to zero (see a discussion in Gajewski, 1993). At parabolic points the stationary-phase solution (A-4) is invalid since its denominator goes to zero and the amplitude becomes infinite. This deficiency is common for all high-frequency ray-theory solutions, including those presented by Ben-Menahem et al. (1991) and Gajewski (1993). Note that the numerical method based on evaluation of FourierBessel integrals (TC90) remains valid even at parabolic points.

For even larger $\sigma, \mathrm{SV}$-wavefronts exhibit cusps centered near phase-velocity maxima. The distribution of energy within the cusps is more complicated than just a single amplitude maximum as in the models considered above, and may not be adequately described by the stationary-phase expression (A-4).

As demonstrated by Figures 10 and 11, the error of the weak-anisotropy approximation (19) in the angular range $0-40^{\circ}$ does not exceed 10 percent if $\sigma<0.2-0.25$. Note that the weak-anisotropy formula is exact in the symmetry direction where the anisotropic correction factor is given by $1 /(1+2 \sigma)$. In a more limited range $0-30^{\circ}$, the weak-anisotropy result remains close to the exact amplitude for much higher values of $\sigma$ (see the model with $\sigma=0.45$ ). However, for $\sigma=0.45$ the weak-anisotropy approximation breaks down completely for group angles over $40^{\circ}$, mostly due to the 
$\sigma=0.3 \quad(\varepsilon=0.15, \delta=0.05)$

SV-Wave Amplitude

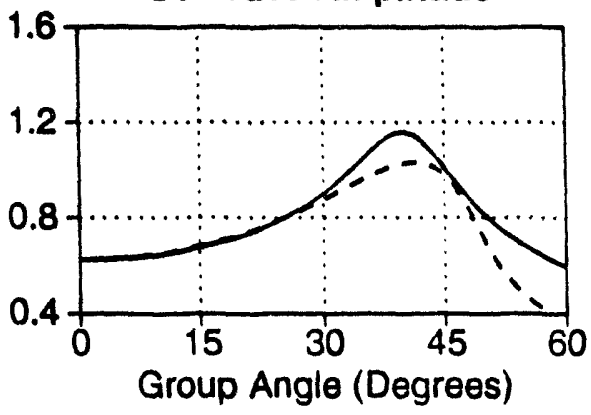

SV-Wave Phase Velocity

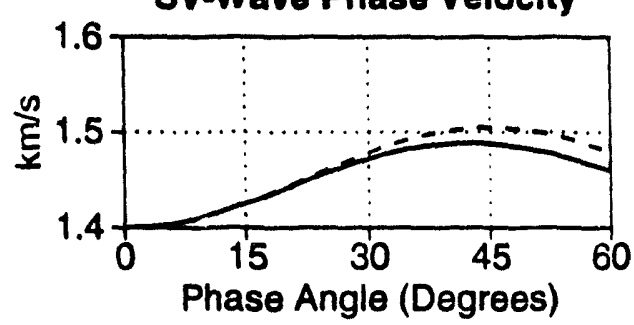

$\sigma=0.45 \quad(\varepsilon=0.2, \delta=0.05)$

SV-Wave Amplitude

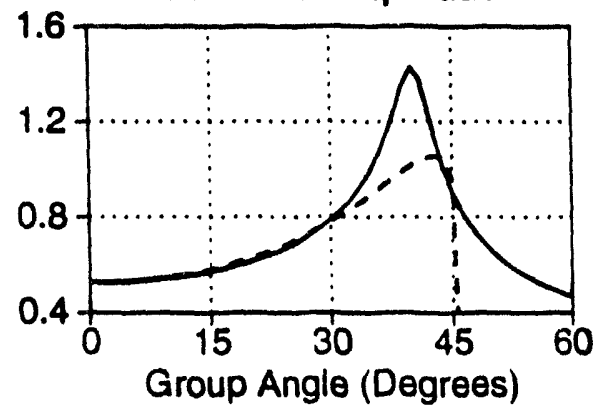

SV-Wave Phase Velocity

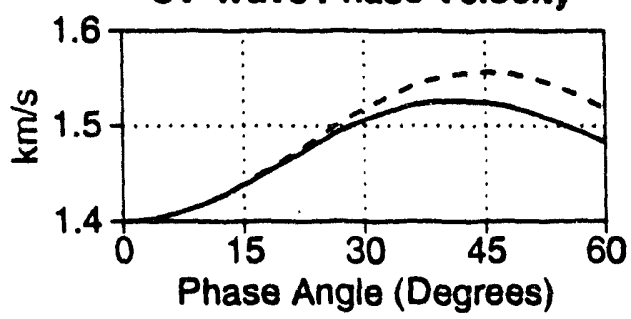

Fig. 11. SV-wave amplitude from a horizontal force for two VTI models with $V_{S 0}=1.4 \mathrm{~km} / \mathrm{s}$; the anisotropic parameters are shown on the plot.

inaccuracy in the group-angle expression (20).

Large magnitude of distortions of SV amplitudes, even in models with moderate velocity anisotropy, means that AVO for SV-waves would be impossible to carry out without an elaborate correction for propagation phenomena. Such correction, however, requires accurate values of the anisotropic coefficients. especially the effective parameter $\sigma$.

\section{SH-wave}

The SH-wave slowness surface in a homogeneous transversely isotropic medium is elliptical, and the phase velocity is given (exactly) by

$$
V_{S H}(\theta)=V_{S 0} \sqrt{1+2 \gamma \sin ^{2} \theta} .
$$

As discussed above, for elliptical anisotropy the far-field solution (A-4) reduces to a concise formula without using the weak-anisotropy approximation. For the SH-wave, equation (A-4) yields:

$$
U_{S H}(R, \psi)=\frac{F_{2}}{4 \pi \rho V_{S 0}^{2} R} \frac{1}{\sqrt{(1+2 \gamma)\left(1+2 \gamma \cos ^{2} \psi\right)}},
$$


$\psi$ is the group angle, $F_{2}$ is the force component perpendicular to the incidence plane. Equation (21) coincides with the formula obtained by Ben-Menahem (1990) in a different way.

The SH-wave radiation pattern (21) is practically identical to the $\mathrm{P}$-wave pattern for elliptical anisotropy $\left(\epsilon=\delta\right.$, equation [13]), just $V_{S O}$ and $\gamma$ should be replaced by $V_{P 0}$ and $\delta$ respectively. The only difference between the two expressions is that the source term $F_{2}$ for the $\mathrm{SH}$-wave is constant because the polarization direction does not change with incidence angle.

For weak SH-wave velocity anisotropy $(\gamma \ll 1)$, equation $(21)$ becomes

$$
U_{S H}(R, \psi)=\frac{F_{2}}{4 \pi \rho V_{S 0}^{2} R} \frac{1+\gamma \sin ^{2} \psi}{1+2 \gamma} .
$$

Again, expression (22) has the same form as the weak-anisotropy approximation for the P-wave (10) in elliptically anisotropic media.

If $\gamma$ is positive (which is usually the case; see Thomsen, 1986), the defocusing of energy at vertical incidence leads to an increase in the SH-wave amplitude with angle. However, as for the P-wave in elliptically anisotropic media, distortions of $\mathrm{SH}$ radiation patterns are relatively mild. For instance, even for the large $\gamma=0.3$ (yielding about 26 percent of the SH-wave velocity anisotropy), the amplitude increase from $0^{\circ}$ to $45^{\circ}$ is limited just to 11 percent. This means that in this angular range the anisotropy-induced directivity in the $\mathrm{SH}$-wave radiation is weak, and the $\mathrm{SH}$ radiation pattern remains close to the isotropic one. The absolute change in the amplitude due to the presence of anisotropy (determined by the term $1 /(1+2 \gamma)$ at normal incidence) is much more pronounced, but this is a different issue.

The conclusion that the influence of transverse isotropy on the shape of SV radiation patterns is much more significant than that on $\mathrm{SH}$ patterns is in good agreement with the experimental results by Robertson and Corrigan (1983), who measured shear-wave radiation patterns in anisotropic shales with positive $\sigma$. They have found a strong focusing of energy of the SV-wave near the $45^{\circ}$ incidence angle, while the $\mathrm{SH}$ radiation pattern did not deviate much from the isotropic one, in spite of a significant degree of velocity anisotropy for the SH-wave.

\section{DISCUSSION AND CONCLUSIONS}

Since conventional methods used to compensate for propagation phenomena in AVO analysis are based on the assumption of isotropy, the influence of anisotropy on the wave propagation to and from the reflector may have a direct influence on the character of AVO anomalies. If not properly corrected for, the anisotropic directivity factor may lead to a change or even a sign reversal in the AVO gradient that is conventionally used in hydrocarbon detection.

Here, the relation between $\mathrm{P}$ - and $\mathrm{S}$-wave radiation patterns and AVO response has been analyzed for transversely isotropic models. A concise analytic solution, obtained 
in the weak-anisotropy approximation, relates the angular dependence of body-wave amplitudes to the anisotropic parameters $\epsilon, \delta$ (for the P-wave), $\sigma$ (SV-wave), and $\gamma$ (SH-wave). Combined with the Thomsen's (1 $\left.r^{\prime} 3\right)$ approximation for the reflection coefficients in transversely isotropic media, $\mathrm{tb}^{\prime}$. solution provides a framework for a comprehensive qualitative analysis of the influence of transverse isotropy on AVO. All analytic expressions in this paper are derived as a function of the phase or group angles with the symmetry axis and. therefore, can be easily applied not only for VTI media, but also for transverse isotropy with any orientation of the axis of symmetry.

The character of the $\mathrm{P}$-wave angular amplitude variations in the range of angles commonly used in AVO analysis $\left(0-40^{\circ}\right)$ is mostly controlled by the difference between the anisotropies $\epsilon$ and $\delta$. and is practically independent of the shear-wave vertical velocity $V_{S 0}$. For models with $\epsilon-\delta>0$, believed to be typical for subsurface formations, transverse isotropy may cause the $P$-wave amplitude to drop by 30 percent and more over the first $40^{\circ}$ from vertical. If the difference $\epsilon-\delta$ is positive, the influence of the anisotropy becomes stronger with increasing $\epsilon-\delta$ and, for fixed $\epsilon-\delta$, with decreasing values of $\epsilon$ and $\delta$. Thus. there is no simple relation between the strength of the velocity anisotropy and the amplitude anomalies.

For elliptical anisotropy $(\epsilon=\delta)$, the distortions of the angular amplitude dependence are well-correlated with the velocity variations. For positive $\epsilon=\delta$, the anisotropy leads to an increase in the amplitude with angle, but these distortions in the $0-40^{\circ}$ angular range are mild, even for models with significant velocity anisotropy. These results show that application of the elliptical-anisotropy approximation $(\epsilon=\delta)$ to $\mathrm{P}$-wave amplitudes may lead to unacceptable errors even if the medium is relatively close to elliptical. It is interesting that the same conclusion applies to the dip-moveout error in transversely isotropic media (Tsvankin, 1994). Since the AVO and dip-moveout problems are principally different (the dip-moveout correction studied by Tsvankin (1994) is purely kinematic), it is likely that the high sensitivity to deviations from the elliptical model is typical for many exploration applications.

The weak-anisotropy approximation for $\mathrm{P}$-wave radiation (10) is more accurate for models with $\epsilon \leq \delta$ than for media with positive $\epsilon-\delta$. If $\epsilon-\delta>0$, the weak-anisotropy result tends to overstate the angular amplitude variations caused by the anisotropy, with the error becoming higher for larger $\epsilon-\delta$. For models with $0<\epsilon-\delta<0.2$ (believed to be most typical), the accuracy of the weak-anisotropy approximation is sufficiently high: for example. the difference between the weak-anisotropy and exact amplitudes in the angular range $0-40^{\circ}$ remains within 10 percent, even if $\epsilon$ reaches 0.25 . The main importance of the weak-anisotropy formula. however, is in providing a convenient tool for qualitative amplitude estimates for a wide range of transversely isotropic models.

The redistribution of energy along the wavefront in TI media is even more significant for the SV-wave than for the $\mathrm{P}$-wave. The influence of transverse isotropy on $\mathrm{SV}$-wave radiation is mostly determined by the effective parameter $\sigma$ introduced by Tsvankin and Thomsen (1994). In a typical model with $\sigma=0.3(\epsilon-\delta=0.1)$ that 
has just 6.3 percent of SV-velocity anisotropy, the SV-wave amplitude increases by 86 percent between $0^{\circ}$ and $40^{\circ}$. For the SH-wave the anisotropy is elliptical, and the distortions of the angular amplitude dependence are relatively mild, even for substantial velocity variations. These results agree with those in the case study by Robertson and Corrigan (1983).

Although here I have examined the influence of radiation patterns on AVO only for VTI models, the far-field solution from TC90 can be applied in azimuthally anisotropic media as well. As shown in TC90, azimuthal anisotropy may significantly reinforce distortions of radiation patterns, even within symmetry planes (e.g., in orthorhombic media). Also, body-wave amplitudes may be significantly disturbed by shear-wave point singularities (Crampin, 1991).

It should be emphasized that wavefront focusing and defocusing phenomena happen not only in the source layer but in any anisotropic layer along the raypath. These distortions may be of equal or greater importance to AVO analysis than the influence of anisotropy on the reflection coefficient, especially for strong reflectors or small differences in the anisotropic coefficients across the reflector.

An approximate correction for simple models such as the one considered in this work can be made by using asymptotic expressions for radiation patterns such as those discussed in this paper or presented by Gajewski (1993). However, the redistribution of energy along the wavefront may occur not only in the source layer but also in any anisotropic layer between the reflector and the surface. The correction for more complicated, realistic models requires application of numerical methods capable of allowing for anisotropy in the wave propagation through layered media.

Therefore, accurate interpretation of AVO anomalies in anisotropic media requires a proper correction for the influence of anisotropy on the wave propagation above the reflector. It is also clear that this correction should be included in any algorithm designed to use reflection coefficients to invert for the anisotropic parameters. If the overburden is laterally homogeneous, it is possible to correct for the propagation effects by doing amplitude calibration at well locations (J. Castagna, pers. comm.). However, such a correction allows one to recover only lateral variations in the reflection coefficient unless it is possible to obtain the reflection coefficient itself from, say, rock properties measurements. However, it is difficult to find the reflection coefficient without doing a correction for propagation phenomena if the medium immediately above the reflector is anisotropic. Also, in this case interpretation of lateral variations in the reflection coefficient is much more complicated. And, clearly, any lateral changes in the overburden make this calibration inaccurate.

Still, the greatest challenge in correcting AVO signatures for anisotropy is to determine the anisotropic parameters with sufficient accuracy. The lowest-order correction to the reflection coefficient depends just on the value of $\delta$ above and below the reflector. For VTI media, $\delta$ can be determined in a straightforward way from the $\mathrm{P}$-wave moveout velocity and the true vertical velocity, if well logs or check shots are available. However, an accurate correction for the propagation phenomena requires 
knowledge not only of $\delta$, but also of $\epsilon$, the parameter that is much more difficult to obtain. Some ways to recover $\epsilon$ are discussed by Tsvankin (1994) and Tsvankin and Thomsen (1995).

\section{ACKNOWLEDGMENTS}

I wish to thank L. Thomsen (Amoco), D. Gajewski (Institute of Geophysics, Hamburg), J. Castagna and D. Foster (ARCO) for useful discussions, K. Larner (CSM) for his thorough review of the manuscript, and T. Galikeev (CSM) for help with software. The support for this work was provided by the members of the Consortium Project on Seismic Inverse Methods for Complex Structures at the Center for Wave Phenomena, Colorado School of Mines, and by the United States Department of Energy, Grant Number DE-FG02-89ER14079 (this support does not constitute an endorsement by DOE of the views expressed in this paper).

\section{REFERENCES}

Aki, K., and Richards, P.G., 1980, Quantitative seismology: theory and methods: W.N. Freeman \& Co., San Francisco.

Banik, N.C., 1987, An effective parameter in transversely isotropic media: Geophysics, 52, 1654-1664.

Ben-Menahem, A., 1990, SH waves from point sources in anisotropic inhomogeneous media: Geophysics, 55, 488-491.

Ben-Menahem, A., Gibson Jr., R.L., and Sena, A.G., 1991, Green's tensor and radiation patterns of point sources in general anisotropic inhomogeneous elastic media: Geophys. J. Int., 107, 297-308.

Berryman, J.G., 1979, Long-wave elastic anisotropy in transversely isotropic media: Geophysics, 44, 896-917.

Crampin, S., 1991, Effects of singularities on shear-wave propagation in sedimentary basins: Geophys. J. Int., 107, 531-543.

Duren, R.E., 1992, Range-equation weights for AVO: Geophysics, 57, 1203-1208.

Gajewski, D., 1993, Radiation from point sources in general anisotropic media: Geophys. J. Int., in press.

Keith, C.M., and Crampin, S., 1977, Seismic body waves in anisotropic media: Reflection and refraction at a plane interface: Geophys. J. R. Astr. Soc., 49, 181-208.

Kim, K.Y., Wrolstad, K.H., and Aminzadeh, F., 1993, Effects of transverse isotropy on P-wave AVO for gas sands: Geophysics, 58, 883-888.

Martinez, R.D., 1993, Wave propagation effects on amplitude variation with offset measurements: A modeling study: Geophysics, 58, 534-543.

Robertson, J.D., and Corrigan, D., 1983, Radiation patterns of a shear-wave vibrator in a near-surface shale: Geophysics, 48, 19-26. 
Samec, P., and Blangy, J.P., 1992, Viscoelastic attenuation, anisotropy, and AVO: Geophysics, 57, 441-450.

Sams, M.S., Worthington, M.H., King, M.S., and Shams Khanshir, M, 1993, A comparison of laboratory and field measurements of P-wave anisotropy: Geophysical Prospecting, 41, 189-206.

Thomsen, L., 1986, Weak elastic anisotropy: Geophysics, 51, 1954-1966.

Thomsen, L., 1993, Weak anisotropic reflections: In Offset Dependent Reflectivity (Castagua and Backus, Eds.), SEG, Tulsa, 103-114.

Tsvankin, I., 1994, Analytic description of dip moveout in anisotropic media: Geophysics, in press.

Tsvankin, I., and Chesnokov, E., 1990a, Synthesis of body-wave seismograms from point sources in anisotropic media: J. Geophys. Res., 95(B7), 11317-11331.

Tsvankin, I., and Chesnokov, E., 1990b, Synthetic waveforms and polarizations at the free surface of an anisotropic halfspace: Geophys. J. Int., 101, 497-505.

Tsvankin, I., and Thomsen, L., 1994, Nonhyperbolic reflection moveout in anisotropic media: Geophysics, in press.

Tsvankin, I., and Thomsen, L., 1995, Inversion of reflection traveltimes for transverse isotropy: Geophysics, submitted.

White, J.E., Martineau-Nicoletis, L., and Monash, C., 1983, Measured anisotropy in Pierre shale: Geophys. Prosp., 31, 709-725.

Wright, J., 1987, The effects of transverse isotropy on reflection amplitude vs offset: Geophysics, 52, 564-567.

\section{APPENDIX A: WEAK-ANISOTROPY APPROXIMATION FOR RADIATION PATTERNS IN TRANSVERSELY ISOTROPIC MEDIA}

In TC90, point-source radiation in homogeneous arbitrary-anisotropic media was decomposed into a Weyl-type integral over plane waves. The displacement from a point force in the frequency domain was shown to be

$$
\vec{U}=\frac{i \omega}{4 \pi^{2}} \sum_{\nu=1}^{3} \int_{-\infty}^{+\infty} \int_{-\infty}^{+\infty} \vec{U}_{p l} e^{-i \omega\left(m_{1} x_{1}+m_{2} x_{2}+m_{3 \nu} x_{3}\right)} d m_{1} d m_{2}
$$

with the plane-wave displacement $\vec{U}_{p l}$ given by

$$
\vec{U}_{p l}=\operatorname{Res}\left[\frac{1}{D\left(m_{3}\right)} G^{a d} \vec{F}\right]_{m_{3 \nu}} .
$$

The source is located at origin of a Cartesian coordinate system. The summation over $\nu$ corresponds to three possible wave types (P- and two S-waves); $\vec{m}$ is the slowness vector, $G_{i k}=c_{i j k l} m_{j} m_{l}-\rho \delta_{i k}$ is the Christoffel matrix, $G^{a d}$ is the adjoint 
matrix of $G, m_{3 \nu}$ (the vertical slownesses of the plane waves) are roots of $D\left(m_{3}\right)=$ $\operatorname{det} G=0$, and $\vec{F}$ is the point-force vector. To obtain the solution in the time domain, (A-1) should be multiplied with the frequency spectrum of the source pulso and substituted into the inverse Fourier transform.

It is convenient to represent $(A-1)$ in the polar ccordinates $\left(m_{1}=m_{0} \cos \phi, m_{2}=\right.$ $\left.m_{0} \sin \phi\right)$ :

$$
\vec{U}=\frac{i \omega}{(2 \pi)^{2}} \sum_{\nu=1}^{3} \int_{0}^{+\infty} \int_{0}^{2 \pi} \vec{U}_{p l} e^{-i \omega\left(\operatorname{mor} \cos (\phi-\alpha)+m_{s \nu} z\right)} m_{0} d m_{0} d \phi,
$$

where $x_{1}=r \cos \alpha, x_{2}=r \sin \alpha, x_{3}=z$.

In TC90, the radiation pattern was derived from (A-3) by means of the stationaryphase approximation valid in the far-field. For the special case of azimuthally-isotropic media the stationary-phase point lies in the incidence plane $\phi=\alpha$, and the phase velocity depends only on the angle with the vertical (symmetry) axis. Then the general stationary-phase solution for the amplitude of the $\nu$-th wave (for brevity, henceforth I omit the superscript $\nu$ ) given in TC90 becomes

$$
\begin{aligned}
&|\vec{U}|= \frac{|\vec{U}|_{p l}}{2 \pi V} \frac{\sin \theta\left(\frac{\cos \theta}{V}+\frac{d(1 / V)}{d \theta} \sin \theta\right)}{\sqrt{\frac{r \sin \theta}{V} \frac{d^{2} \phi}{d \theta^{2}}}} \\
& \frac{d^{2} \Phi}{d \theta^{2}}=(r \sin \theta+z \cos \theta)\left[\frac{1}{V}-\frac{d^{2}(1 / V)}{d \theta^{2}}\right. \\
&\left.+\frac{2}{V}\left(\frac{r \cos \theta-z \sin \theta}{r \sin \theta+z \cos \theta}\right)^{2}\right]
\end{aligned}
$$

where $\Phi=-m_{0} r-m_{3 \nu} z$ is the frequency-independent part of the phase function in the incidence plane (see equation [A-3]), $\theta$ is the phase angle measured from the vertical (z) axis, $V$ is the phase velocity. Expression (A-4) should be evaluated at the phase angle $\theta$, corresponding to a given ray (group velocity) angle $\psi$ determined by the receiver position $(\tan \psi=r / z)$.

Here, the exact far-field solution for transverse isotropy (A-4) is transformed into a much simpler weak-anisotropy approximation. The derivation is given for the $\mathrm{P}$-wave only; the radiation patterns of S-waves are obtained in a similar way.

In the following, it is assumed that the source and receiver are located in the $\left[x_{1}, x_{3}\right]$ plane; in this case, at the stationary-phase point $\phi=\alpha=0$. It is convenient to represent $\left|\vec{U}_{p l}\right|(\mathrm{A}-2)$ as an explicit function of the $\mathrm{P}$-wave slowness vector. The non-zero components of the Christoffel matrix in the plane $\left[x_{1}, x_{3}\right]$ are

$$
G_{11}=c_{11} m_{0}^{2}+c_{44} m_{3}^{2}-\rho
$$




$$
\begin{gathered}
G_{22}=c_{66} m_{0}^{2}+c_{44} m_{3}^{2}-\rho \\
G_{33}=c_{44} m_{0}^{2}+c_{33} m_{3}^{2}-\rho \\
G_{13}=G_{31}=m_{0} m_{3}\left(c_{13}+c_{44}\right) .
\end{gathered}
$$

The determinant of $\mathrm{G}$ is

$$
D=\operatorname{det} G=G_{22}\left(G_{11} G_{33}-G_{13}^{2}\right)
$$

The vertical slownesses of the $\mathrm{P}$ - and SV-waves are the roots of the polynomial $G_{11} G_{33}-G_{13}^{2}$, while the solutions of $G_{22}=0$ give the vertical slownesses for the SHwave. Since the $\mathrm{P}$-wave displacement vector lies in the $\left[x_{1}, x_{3}\right]$ plane, formula (A-2) for the $\mathrm{P}$-wave yields

$$
\begin{aligned}
& U_{p l 1}=H\left(F_{1} G_{33}-F_{3} G_{13}\right), \\
& U_{p l 3}=H\left(F_{3} G_{11}-F_{1} G_{13}\right),
\end{aligned}
$$

with

$$
H=\left.\frac{m_{3}-m_{3 P}}{\operatorname{det} G}\right|_{m_{3 P}}=\frac{1}{2 c_{33} c_{44} m_{3 P}\left(m_{3 P}^{2}-m_{3 S V}^{2}\right)},
$$

$m_{3 P}$ and $m_{3 S V}$ are the vertical slownesses of the $\mathrm{P}$ - and SV-waves for a fixed $m_{0}$. $m_{3 S V}$ is given by

$$
m_{3 S V}^{2}=\frac{\left(c_{11} m_{0}^{2}-\rho\right)\left(c_{44} m_{0}^{2}-\rho\right)}{c_{33} c_{44} m_{3 P}^{2}}
$$

Taking into account that for $m_{3 P} G_{11} G_{33}-G_{13}^{2}=0$, we find from (A-10) and $(\mathrm{A}-11)$ :

$$
\left|\vec{U}_{p l}\right|=H F_{u}\left(G_{11}+G_{33}\right),
$$

where $F_{u}$ is the projection of the force on the plane-wave displacement vector $\vec{U}_{p l}$.

Substututing $H$ from (A-12) and (A-13), $G_{11}$ from (A-6) and $G_{33}$ from (A-8) into (A-14) yields

$$
\left|\vec{U}_{p l}\right|=\frac{F_{u} m_{3 P}}{2} \frac{m_{0}^{2}\left(c_{11}+c_{44}\right)+m_{3 P}^{2}\left(c_{33}+c_{44}\right)-2 \rho}{m_{3 P}^{4} c_{33} c_{44}-\left(c_{11} m_{0}^{2}-\rho\right)\left(c_{44} m_{0}^{2}-\rho\right)} .
$$


Equation (A-15) is valid for general transverse isotropy. At this point, we substitute $m_{0}=\frac{\sin \theta}{V}, m_{3 P}=\frac{\cos \theta}{V}$, and introduce the weak-anisotropy approximation for the P-wave phase velocity (Thomsen, 1986)

$$
V_{P}(\theta)=V_{P 0}\left(1+\delta \sin ^{2} \theta \cos ^{2} \theta+\epsilon \sin ^{4} \theta\right) .
$$

Formula (A-16) along with the expressions for $\epsilon$ and $\delta$ (equations (1),(2) of the main text) can be used to find the weak-anisotropy approximation for equation (A15). Linearization in $\epsilon$ and $\delta$, after tedious but straightforward algebra, gives

$$
\left|\vec{U}_{p l}\right|=\frac{F_{u}}{2 V_{P}(\theta) \rho \cos \theta}\left[1+2 \sin ^{2} \theta\left(\delta \cos 2 \theta+2 \epsilon \sin ^{2} \theta\right)\right]
$$

The next step is to obtain the weak-anisotropy approximation for $\frac{d^{2} \phi}{d \theta^{2}}(A-5)$. Expressing $r$ and $z$ through the source-receiver distance $R(r=R \sin \psi, z=R \cos \psi)$, we find

$$
r \sin \theta+z \cos \theta=R \cos (\psi-\theta)
$$

and

$$
\frac{r \cos \theta-z \sin \theta}{r \sin \theta+z \cos \theta}=\frac{\tan \psi-\tan \theta}{1+\tan \psi \tan \theta}
$$

The weak-anisotropy approximation for the P-wave group angle $\psi$ is (Thomsen, 1986)

$$
\tan \psi=\tan \theta\left[1+2 \delta+4(\epsilon-\delta) \sin ^{2} \theta\right]
$$

Substituting equations (A-18), (A-19), (A-20) along with the derivative of the phase-velocity function (A-16) into formula (A-5) and dropping the terms quadratic in $\epsilon$ and $\delta$, we get

$$
\frac{d^{2} \Phi}{d \theta^{2}}=\frac{R}{V}\left(1+\frac{1}{V} \frac{d^{2} V}{d \theta^{2}}\right) .
$$

Using the phase-velocity function (A-16) in the numerator of equation (A-4) yields

$$
\frac{\cos \theta}{V}+\frac{d(1 / V)}{d \theta} \sin \theta=\frac{\cos \theta}{V}\left[1-2 \sin ^{2} \theta\left(\delta \cos 2 \theta+2 \epsilon \sin ^{2} \theta\right)\right]
$$

Finally, formulas $(A-17),(A-21)$, and (A-22) are substituted into the original stationary-phase expression $(A-4)$ : 


$$
|\vec{U}|=\frac{F_{u}}{4 \pi \rho V^{2} R} \frac{1}{\sqrt{\frac{\sin \phi}{\sin \theta}\left(1+\frac{1}{V} \frac{d g}{d \rho}\right)}} .
$$

Equation (A-23) can be fully linearized in the anisotropies $\epsilon$ and $\delta$. However, expression (A-23) is a useful intermediate result because analogous derivations lead to the same formula for S-waves. Linearized expressions for each wave type (P, SV, $\mathrm{SH})$ are discussed in the main text. 


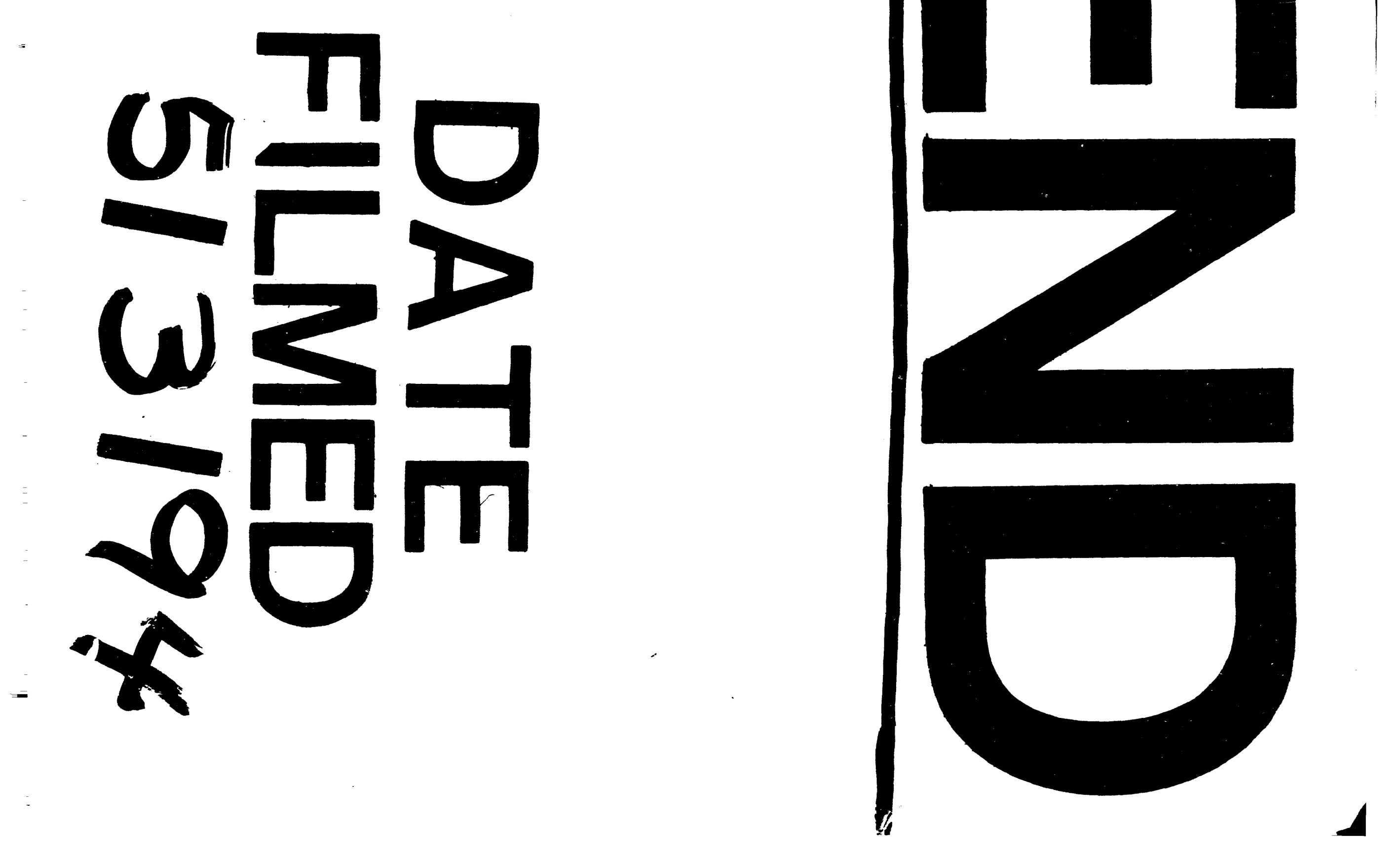


